\title{
Wireless Covert Channel With Polarized Dirty Constellation in Backscatter Communication
}

\section{Sen Qiao}

Nanjing University of Information Science and Technology https://orcid.org/0000-0002-3710-9590

Guangjie Liu ( $\nabla$ gjieliu@gmail.com )

Nanjing University of Information Science and Technology https://orcid.org/0000-0003-4729-7406

Jianfeng Shi

Nanjing University of Information Science and Technology

Xiaopeng Ji

Nanjing University of Information Science and Technology

Weiwei Liu

Nanjing University of Information Science and Technology

\section{Research Article}

Keywords: covert communication, backscatter communication, channel polarization, dirty constellation

Posted Date: August 30th, 2021

DOI: https://doi.org/10.21203/rs.3.rs-816334/v1

License: (c) (1) This work is licensed under a Creative Commons Attribution 4.0 International License. Read Full License 


\title{
RESEARCH
}

\section{Wireless Covert Channel with Polarized Dirty Constellation in Backscatter Communication}

\author{
Sen Qiao ${ }^{1}$, Guangjie Liu ${ }^{1 *}$, Jianfeng Shi ${ }^{1,2}$, Xiaopeng $\mathrm{Ji}^{1}$ and Weiwei Liu ${ }^{3}$
}

${ }^{*}$ Correspondence:
gjieliu@gmail.com
${ }^{1}$ School of Electrical and
Information Engineering, Nanjing
University of Information Science
and Technology, Nanjing, China
Full list of author information is
available at the end of the article

available at the end of the article

\begin{abstract}
Ambient backscatter communication has become a low-cost and green solution to next-generation Internet-of-Things (IoT). In this paper, we investigate a problem of wireless covert communication in backscatter communication system. The purpose of covert communication is to hide the process of communication and avoid being detected by eavesdropper. Covert messages are modulated into a random-like noise, and then superimposed on the carrier signal. Specially, we propose a wireless covert channel with polarization dirty constellation (WCC-PDC), which is polarized to solve the problem of low signal power in backscatter communication. Some methods are used to combine covert code block with carrier information block and increase the undetectability of covert communication. Additionally, successive-cancellation decoder and successive-cancellation list decoder are adopted to improve the reliability of WCC-PDC. Simulation results are finally provided to verify the improvement of undetectability and reliability with WCC-PDC.
\end{abstract}

Keywords: covert communication; backscatter communication; channel polarization; dirty constellation

\section{Introduction}

Backscatter communication powered by ambient or dedicated radio-frequency (RF) sources has drawn increasing attention over the past two decades[1, 2]. A typical backscatter communication system mainly consists of a tag and a reader. Depending on the tag's power supply, backscatter communication systems can be divided into active, passive or semi-passive [3]. In particular, ambient backscatter communication $(\mathrm{AmBC})$ enables backscatter devices (BDs) to transmit information to nearby receivers[4]. Since AmBC exempts the BDs from generating RF carriers, it becomes a low-cost and green solution to next-generation Internet-of-Things(IoT)[5].

Due to the accessibility of wireless channel, privacy and security of wireless communication have been seriously threatened. Sometimes we can improve the security of wireless communication through encryption or encoding. At other times, communication must also be covert, or undetectable which has a low probability of intercept (LPI) or a low probability of detection (LPD)[6]. LPD communication methods are meaningful, especially when the act of communication will raise some concerns. As a branch of modern information hiding technology in the field of LPD communication, covert communication hide the transmission process of secret information. Even if an eavesdropper intercepts the communication signal, it cannot be distinguished from normal wireless communication[7,8]. The methods of covert communication 
include artificial additional signal noise[9], artificial coding domain error[10], insertion of additional signal band, etc. Because of sharing the same spectrum, AmBC system suffers strong interference from the RF source. Some existing research treat the interference as part of background noise[11, 12, 13]. To mask the process of communication, we can "pre-distort" the signal to mimic constellation error arising from the AWGN(Additive White Gaussian Noise) channel[6]. It is the most widely used physical layer wireless covert communication at present.

Despite a lot of research has been done on the security and privacy of backscatter systems, covert communication in backscatter communication has not been widely studied before. In [14], Shahzad has proposed a method of covert communication in a backscatter system. Reader manipulates the radio-frequency $(\mathrm{RF})$ signal such that Willie remains unaware of the tag's response state. Yet, the reliability and undetectability of covert communication are poor. By increasing the transmission power of RF signal, undetectability will deteriorate[15, 16]. While if we map the covert information into Gaussian noise, the undetectability ameliorates with the reliability deteriorates[14, 17]. To improve the reliability and undetectability of covert communication, we plan to design a dedicated radio-frequency source to transmit covert messages in RF signals directly. Tag is a passive node, just reflect the incident wave to Bob.

To increase the undetectability and reliability of covert communication in backscatter system, we propose a wireless covert channel with polarized dirty constellation(WCCPDC) in backscatter communication system. The wireless covert communication is set at the physical layer, we disguise covert information as constellation errors. To solve the problem of low signal power in backscatter communication system, we polarize the covert channel. And we fill the covert code blocks with 0 or 1 bits to accommodate different block lengths. Furthermore, the polarized dirty constellations are rotated to improve the undetectability. By encoding the dirty constellation, we can obtain better reliability and undetectability. Compared with other physical layer covert communication methods, WCC-PDC can obtain better physical realization. The contributions of this paper are summarized as follows:

(1) To increase the undetectability of covert communication in backscatter system, we designed a dedicated radio-frequency source to transmit covert messages in RF signals directly.

(2) We investigated the relationship between undetectability and reliability of covert communication in backscatter system. To increase the reliability and undetectability, we polarized the covert channel and rotated the polarized dirty constellation.

(3) We adopted a successive-cancellation list decoder to improve the performance of the polarized covert channel.

The remainder of this paper is organized as follows. In the next section, system model and wireless covert channel with dirty constellation are introduced. In Section 3 , the basis of our scheme including binary hypothesis testing and polarization dirty constellation are described. In Section 4, WCC-PDC is proposed, where some specific operations are adopted to combine dirty constellation with polar code. Two different decoding algorithms are used to improve the reliability of WCC-PDC. Section 5 presents the experimental results on undetectability and reliability. Finally, Section 6 concludes the whole paper. 


\section{Related work}

\section{System Model}

Similar to the famous Alice-Bob model[18], there is a transmitter, a detector, and a backscatter node, i.e., a Tag and a Reader. We can also call them Alice, Bob, and Willie, as shown in Figure 1. The components of tag include a modulation block, a decoder, a battery, and an RF energy harvesting block. Tag is a passive node, just reflect the incident wave to Bob.

Alice trys to transmit covert information to Bob without being detected by Willie.

Figure 1 The framework of wireless covert communication

Willie observes the wireless channel and detects whether covert communication exists or not. Let the transmit signal as $s(n) e^{j 2 \pi f_{s} n}$. Let $f_{s}$ as carrier frequency, $s(n)$ as complex baseband signal. Binary switch $B(n)$ is used to indicate signal transmission. Specially, if $B(n)=0$, little signal can be reflected, while if $B(n)=1$, the signal can be scattered to the reader. Mathematically, we can express the signal received by tag as

$$
x(n)=h s(n) e^{j 2 \pi f_{s} n}
$$

Some carrier phase offset may exist between $x(n)$ and $s(n)$, which can be absorbed into the complex channel $h$. Moreover, the signal backscattered can be expressed as

$$
a(n)=\eta B(n) x(n)
$$

where $\eta$ is the attenuation of complex signal in the tag. While if we keep $B(n)=1$, the signal can be scattered to the reader continuously. The tag can be used as a relay equivalently. We denote the carrier phase offset between the Alice (i.e., RF source) and Bob (i.e., reader) as $\theta_{0}$. The signal received by Bob can be expressed as

$$
\tilde{y}(n)=\eta g h s(n) e^{j 2 \pi f_{s} n+\theta_{0}}+\sigma(n)_{w}^{2}
$$

where $\sigma(n)_{w}^{2}$ is additive white Gaussian noise in the channel, $g$ is the channel between tag and Bob. The main task of Bob is to recover $s(n) e^{j 2 \pi f_{s} n}$ from $y(n)$. The main task of Willie is to observe the channel and detect whether covert communication exists or not.

Willie's probability of detection error consists of two components: the probability of missed detection $P_{F}$ and the probability of false alarm $P_{M}$. Considering the actual detect condition, Willie can only detect finite samples. It is numerically shown that with noise uncertainty at Willie there may exist an optimal number of samples that maximizes the communication rate subject to $\xi \geq 1-\varepsilon$, where $\xi$ is the sum of $P_{F}$ (i.e., false alarm rate) and $P_{M}$ ( i.e., miss detection rate) at Willie. Let an arbitrarily small number $\varepsilon$ as the maximum acceptable detection rate of Willie. 
Wireless covert channel with dirty constellation

To reduce the probability of covert information being detected by Willie, covert messages can be converted to constellation errors of common signal, which is also called wireless covert communication with dirty constellation (WCC-DC).

Figure 2 (a)The framework of wireless covert communication with dirty constellation(b)Rotation of Covert Constellation in WCC-DC

The framework of WCC-DC is illustrated in Figure 2 (a). The premise of WCCDC is wireless communication physical layer with OFDM structure. In order to reduce the statistical correlation, transmitter can divide all OFDM subcarrier into secret subcarriers or normal subcarriers. The corresponding mapping sequence are also generated. The secret information will be modulated with QPSK to obtain covert signal. Covert signal and random noise signal are superposed on the subcarrier signal. We denote the power ratio between carrier signal and covert signal as carrier covert ratio $(C S R)$. To ameliorate the undetectability of covert communication, the covert constellation will be rotated at a certain angle, as shown in Figure 2(b). The transmitter and receiver should share the mapping sequence before the covert communication starts. The receiver should demodulate the carrier message as normal first. Then, the carrier message should be modulated again to obtain ideal carrier signal. The receiver subtract ideal carrier signal from received signal to get residual signal, which is the covert signal with noise. The covert signal will be rotated in the opposite direction at certain angle and demodulated with QPSK. The covert message will be extracted from modulated signal according to the mapping sequence.

Let the average transmit power of Alice as $p$. Referring to Eq.3, we can obtain the $S N R$ of transmit signal received by Bob

$$
S N R=\frac{|\eta|^{2}|h|^{2}|g|^{2} p}{\sigma(n)_{w}^{2}}
$$

The power of subcarrier signal is lower than the signal received by tag. The reliability deteriorates with low $S N R$. When we increase the $C S R$, the undetectability will be deteriorated. Therefore, we plan to ameliorate the reliability of covert message at low $C S R$ with polar codes.

\section{Methods}

\section{Binary Hypothesis Testing at Willie}

According to the system model shown in Figure 1, the performance elements of the wireless covert channel mainly include two aspects: undetectability and reliability. In communication, Alice totally transmits $n$ symbols to Bob. We denote the finite block as $m[i](i \in[1, n])$, which consist of normal information $m_{c}[i]$ and secret information $m_{s}[i]$. William's task is to collect the messages $m[i]$ transmitted by Alice and detect whether covert communication exists or not. Let the AWGN(Additive White Gaussian Noise) between Alice and Bob as $r[i] \sim\left(0, \sigma_{w}^{2}\right)$. The signal received 
by Willie can be expressed as

$$
y_{w}[i]=m[i]+r[i]
$$

The task of Willie is to detect whether covert communication exists. We define two hypotheses, $H_{0}$ and $H_{1}$, to distinguish these two cases:

$$
\left\{\begin{array}{l}
H_{0}: m[i]=m_{c}[i] \\
H_{1}: m[i]=m_{c}[i]+m_{s}[i]
\end{array}\right.
$$

Let $H_{0}$ denotes that covert communication exists, $H_{1}$ denotes covert communication does not exist. In covert communication, the ultimate goal of Willie is to minimize the total error rate (i.e., $\xi$ ). Let $T$ and $F$ denote binary decisions that infer whether covert communication exists or not. The false alarm rate and miss detection rate can be expressed as

$$
\left\{\begin{array}{l}
P_{F}=P_{r}\left(T \mid H_{0}\right) \\
P_{M}=P_{r}\left(F \mid H_{1}\right)
\end{array}\right.
$$

Assuming Willies perform the optimal detect. Following Pinsker's inequality [19, 20], we can obtain

$$
P_{F}+P_{M} \geq 1-\sqrt{\frac{1}{2} D\left(P_{0} \| P_{1}\right)}
$$

where relative entropy $D\left(P_{0} \| P_{1}\right)$ (also called $\mathrm{K}-\mathrm{L}$ divergence) is defined as follows:

$$
D\left(P_{0} \| P_{1}\right)=\int_{n} p_{0}(x) \ln \frac{p_{0}(x)}{p_{1}(x)} d x
$$

where $n$ is the value range of $x$. Let $p_{0}(x) \sim\left(0, \sigma_{s}\right) \sim\left(0, P+\sigma_{\omega}^{2}\right)$ denotes the distribution of sequence $P_{0}$ detected by Willie, and $p_{1}(x) \sim\left(0, \sigma_{\omega}\right)$ denotes the distribution of AWGN $P_{1}$ collected by Willie. Let $P$ denotes the transmit power. We can equivalently express Eq.9 as

$$
\begin{aligned}
& D\left(P_{0} \| P_{1}\right)=\int_{n} \frac{1}{\sqrt{2 \pi} \sigma_{\omega}} e^{-\frac{x^{2}}{\sigma_{\omega}^{2}}} \cdot \ln \left(\frac{\sigma_{s}}{\sigma_{\omega}} \cdot e^{\frac{x^{2}}{\sigma_{\omega}^{2}}-\frac{x^{2}}{\sigma_{s}^{2}}}\right) d x \\
& =\int_{n} \frac{1}{\sqrt{2 \pi} \sigma_{\omega}} e^{-\frac{x^{2}}{\sigma_{\omega}^{2}}} \cdot\left(\ln \left(\frac{\sqrt{P+\sigma_{\omega}^{2}}}{\sigma_{\omega}}\right)+\frac{x^{2} \cdot P}{\left(P+\sigma_{\omega}^{2}\right) \cdot \sigma_{\omega}^{2}}\right) d x \\
& =\frac{1}{2}\left(\ln \frac{P+\sigma_{\omega}^{2}}{\sigma_{\omega}^{2}}-\frac{P}{P+\sigma_{\omega}^{2}}\right)
\end{aligned}
$$

It can be obtained that the $\mathrm{K}-\mathrm{L}$ divergence deteriorates with the increase of transmit power $P$. We can ameliorate the value of $\mathrm{K}-\mathrm{L}$ divergence by constraining the transmit power. Except KL-divergence, we can also calculate the distance between distributions with KS-distance (also called Kolmogorov-Smirnov statistic). The KSdistance [21] is defined as follows:

$$
D_{K S}=\max \left|F_{1}(x)-F_{0}(x)\right|
$$


where $F_{1}(x)=\frac{\sum_{j=1}^{x} h_{1}(j)}{n}, F_{0}(x)=\frac{\sum_{j=1}^{x} h_{0}(j)}{n}$. Let $F_{1}(x)$ and $F_{0}(x)$ denote the cumulative distribution functions of $P_{1}$ and $P_{0} . P_{1}$ and $P_{0}$ are both divided into $K$ bins. The number of the elements in $P_{1}$ and $P_{0}$ are denoted by $h_{1}(j)$ and $h_{0}(j)$, $j \in(1,2 \cdots K)$. We can expressed Eq.11 equivalently as

$$
\begin{aligned}
& D_{K S}=\max \left|\operatorname{erf}\left(\frac{x}{\sigma_{\omega}}\right)-\operatorname{erf}\left(\frac{x}{\sigma_{s}}\right)\right| \\
& =\max \left|\operatorname{erf}\left(\frac{x}{\sigma_{\omega}}\right)-\operatorname{erf}\left(\frac{x}{\sqrt{\sigma_{\omega}^{2}+P}}\right)\right|
\end{aligned}
$$

It can be obtained that we can also ameliorate the value of KS-distance by constraining the transmit power. In summary, we can improve the undetectability by constraining the power of covert communication. However, the BER (Bit Error Rate) would deteriorate with low transmit power. Therefore, channel coding is a feasible solution to ameliorate the reliability of covert communication.

\section{Dirty Constellation Polarization}

We always denote a binary discrete memoryless channel (B-DMC) $W$ as $W: X \rightarrow Y$ with input alphabet $X$ and output alphabet $Y$. The input alphabet $X$ belongs to $\operatorname{set}\{0,1\}$, the output alphabet $Y$ may be arbitrary. To measure the channel condition, Arıkan [22] mainly used two parameters: the symmetric capacity

$$
I(W) \triangleq \sum_{y \in Y} \sum_{x \in X} \frac{1}{2} W(y \mid x) \log \frac{W(y \mid x)}{\frac{1}{2} W(y \mid 0)+\frac{1}{2} W(y \mid 1)}
$$

and the Bhattacharyya parameter.

$$
Z(W) \triangleq \sum_{y \in Y} \sqrt{W(y \mid 0) W(y \mid 1)}
$$

When the input alphabet is just 1 or $0, Z(W)$ is an upper bound of the error probability of maximum-likelihood (ML) decision. It is easy to see that $Z(W)$ and $I(W)$ both take values in $[0,1]$. Intuitively, we will expect $I(W) \approx 1$ iff $Z(W) \approx 0$ or $I(W) \approx 0$ iff $Z(W) \approx 1$.

In [23], Gallager proposed that the ensemble average probability of decoding error basing on ML decoding satisfies

$$
\bar{P}_{e} \leq \exp \left\{-N\left[E_{0}(\rho, Q)-\rho R\right]\right\}
$$

where

$$
E_{0}(\rho, Q)=-\ln \sum_{j=0}^{J-1}\left[\sum_{k=0}^{K-1} Q(k) P(j \mid k)^{1 /(1+\rho)}\right]^{1+\rho}
$$

We denote the transition probability of discrete memoryless channel as $P(j \mid k)$, positive integer as $N$ and positive number as $R$. In the ensemble of $(N, R)$ block 
codes, every code is independently chosen with the probability assignment $Q(k)$. Assuming $\rho=1$, we can transform Eq.16 as

$$
E_{0}(1, Q)=-\ln \sum_{j=0}^{J-1}\left[\sum_{k=0}^{K-1} Q(k) P(j \mid k)^{1 / 2}\right]^{2}
$$

For binary symmetric channel, $E_{0}(1, Q)$ is maximized over $Q$ by $Q(0)=Q(1)=1 / 2$. For this $Q$, we have

$$
\begin{aligned}
& E_{0}(1, Q)=\ln 2-2 \ln \left[p^{1 / 2}+(1-p)^{1 / 2}\right] \\
& =\ln \frac{2}{\left(p^{1 / 2}+(1-p)^{1 / 2}\right)^{2}} \\
& =\ln \frac{2}{1+Z(W)}
\end{aligned}
$$

The Eq.15 can be converted to

$$
N R \geq \ln \left(\bar{P}_{e}\right)+N \cdot \ln \frac{2}{1+Z(W)} \sim \log \left(\bar{P}_{e}\right)+N \cdot \log \frac{2}{1+Z(W)}
$$

$R$ in nats (that is, using $\ln$ ) is the number of binary digits entering the encoder per transmitted per second. Thus we use $R$ in bits (that is, using $\log 2$ ) to interpret the Eq.19, which is the number of binary digits entering the encoder per transmitted channel digit. It is known that $I(W)=C \geq R$, so we have

$$
I(W) \geq \log \frac{2}{1+Z(W)}
$$

For any B-DMC, we have

$$
\left\{\begin{array}{l}
I(W) \geq \log \frac{2}{1+Z(W)} \\
I(W) \leq \sqrt{1-Z(W)^{2}}
\end{array}\right.
$$

When $W$ is a symmetric channel, the symmetric capacity of $W$ equal to Shannon capacity. Refer to Eq.17, it can be obtained that $I(W)$ approaching 1 when $Z(W) \approx 0$. It shows that there exists a sequence of $\left(2^{N R}, N\right)$ codes with maximum probability of error $P_{e} \rightarrow 0$ when we encode the covert information in B-DMC. Polarization code can realize $P_{e} \rightarrow 0$ with polarizing the channel. Channel polarization is an operation consisting of channel combining and channel splitting. With the length of block $N$ goes to infinity, the symmetric capacity $I(W)$ tends to two polar. Part of the symmetric capacity tends to 1 , the rest tends to 0 . If we polarize the covert channel in B-DMC, as the length of covert information block $N$ becomes large, part of covert information can be transmitted with Shannon capacity. We can improve the undetectability by reducing the power of the covert signal without deteriorating the reliability of covert communication.

If the communication channel is AWGN channel, the transmit process of covert 
information can be expressed as $X_{1}^{N} \rightarrow S_{1}^{N} \rightarrow Y_{1}^{N}$. The signal $Y_{1}^{N}$ received by Bob can be expressed as $y_{i}=s_{i}+n_{i}, 1 \leq i \leq N$. We denote the encoded covert information as $S_{1}^{N}$. Assuming that each $X_{1}^{N}$ has the same probability, the probability of each $X_{1}^{N}$ is $2^{-N R}$. We denote the BER (Bit Error Rate) basing on maximum likelihood decoding as $P_{e}^{M L}$. The UUB (Union Upper Bound) of BLER (Block Error Rate) basing on maximum likelihood decoding can be written as $P_{e}^{M L, U U B}$ with

$$
\begin{aligned}
& P_{e}^{M L} \leq P_{e}^{M L, U U B}=\sum_{x \in X} \operatorname{Pr}(x) P_{e}^{(N)}(x) \\
& =\frac{1}{2^{N R}} \sum_{x \in X} \operatorname{Pr}(x) \sum_{w=1}^{2^{N R}} \lambda_{w}(C) \\
& =\sum_{d=d_{1}}^{d_{N R}} A_{d} Q\left(\frac{\sqrt{d}}{\sigma}\right) \\
& =\sum_{d=d_{1}}^{d_{N R}} A_{d} Q\left(\sqrt{\frac{2 R d E_{b}}{N_{0}}}\right)
\end{aligned}
$$

We use the standard notation $P_{e}^{(N)}(x), \lambda_{w}(C)$ to denote the probability of error. We denote the Hamming distance and the codes with the same Hamming distance as $d, A_{d}$.

\section{Wireless covert channel with Polarized Dirty Constellation}

To ameliorate the undetectability and reliability of WCC-DC, we proposed wireless covert communication with polarized dirty constellation (WCC-PDC). The schematic diagram of polarized dirty constellation is illustrated in Figure 3. Parts of

Figure 3 The schematic diagram of wireless covert communication with polarization dirty constellation

the framework of WCC-PDC will be illustrated specially in the following chapters.

\section{Polar Encoding}

We use the standard notation $x_{1}^{N}=u_{1}^{N} \cdot G_{N}$ to denote the encoding operation. $G_{N}$ is the conversion matrix consisting of various permutation operations. Erdal Arıkan[] provides a recursive formula as

$$
\begin{aligned}
& G_{N}=R_{N}\left(F \otimes I_{N / 2}\right)\left(I_{2} \otimes G_{N / 2}\right) \\
& =R_{N}\left(F \otimes G_{N / 2}\right)
\end{aligned}
$$

Let $I_{k}$ denote the $k$-dimensional identity matrix for any positive integer $k$. We assume $N=2^{n}$ for positive integer $n$. It is simple to derive the recursive relationship as

$$
G_{N}=R_{N}\left[F \otimes\left(R_{N / 2}\left(F \otimes G_{N / 4}\right)\right)\right.
$$


With the identity $A C \otimes B D=(A \otimes B)(C \otimes D)$, we can equivalently rewrite Eq.20 as

$$
\begin{aligned}
& G_{N}=R_{N}\left(I_{2} \otimes R_{N / 2}\right)\left(F^{\otimes 2} \otimes G_{N / 4}\right) \\
& =R_{N}\left(I_{2} \otimes R_{N / 2}\right)\left(I_{4} \otimes R_{N / 4}\right) \cdots\left(I_{N / 2} \otimes R_{2}\right) F^{\otimes n}
\end{aligned}
$$

Where $F^{\otimes n}$ is the n-dimensional Kronecker product of $F$ matrix with $F$ equals $\left[\begin{array}{ll}1 & 0 \\ 1 & 1\end{array}\right] \cdot R_{N}$ is converted from the $N$-dimensional identity matrix, arranging the column vectors of the identity matrix in the order of odd first and then even. We can express $R_{N}$ as

$$
\begin{aligned}
& R_{N}=\left[I_{N, \text { odd }}, I_{N, \text { even }}\right] \\
& =\left[I_{N, 1}, I_{N, 3}, \cdots I_{N, N-1}, I_{N, 2}, I_{N, 4}, \cdots I_{N, N}\right]
\end{aligned}
$$

where $I_{N, x}$ denotes the $x$-th column of matrix $I_{N}$ for positive integer $x \in[1, N]$. Let covert message $u_{1}^{N}$ be any vector over GF (2). We would obtain $x_{1}^{N}=u_{1}^{N} \cdot G_{N}$ at output. Some preprocessing needs to be done to combine polar code and dirty constellation.

Fill and Rotation

The size of the covert information block and the carrier information block are incompatible, the covert information needs to be filled with all 0 or all 1 bits as shown in Fig. 4. For reducing the statistical relevance of covert information, our scheme will rotate the covert constellation point. If we modulate the covert information with BPSK, we see from Figure 4 the position of initial covert constellation points and carrier constellation points. To improve the undetectability, we rotate the covert

Figure 4 Rotation of Polarized Covert Constellation

constellation point with $n \theta$ degree with $n \in\left[1, \frac{\pi}{\theta}\right]$. Let us assume the coordinate of carrier constellation point is $\left(m_{c, r}, m_{c, i}\right)$. And the coordinate of covert constellation point is $\left(m_{c, r}+m_{s, r}, m_{c, r}+m_{s, i}\right)$. When we rotate the covert constellation point, we can obtain

$$
\left\{\begin{array}{l}
\tilde{m}_{s, r}=\cos (n \theta) \cdot m_{s, r}-\sin (n \theta) \cdot m_{s, i} \\
\tilde{m}_{s, i}=\cos (n \theta) \cdot m_{s, i}+\sin (n \theta) \cdot m_{s, r}
\end{array}\right.
$$

As we known, the covert information is modulated with BPSK. Thus, we can express the covert constellation point as

$$
\left\{\begin{array}{l}
\tilde{m}_{s, r}[i]=\cos \left(\left(i-\left\lfloor\frac{i}{n}\right\rfloor \cdot n\right) \theta\right) \cdot m_{s, r}[i] \\
\tilde{m}_{s, i}[i]=\sin \left(\left(i-\left\lfloor\frac{i}{n}\right\rfloor \cdot n\right) \theta\right) \cdot m_{s, r}[i]
\end{array}\right.
$$


When we receive the subcarrier signal, the received carrier information should be modulated again to obtain the residual signal. With reversing the rotation of residual signal, we can obatin the covert signal. The covert information can be obtained by demodulating the covert signal with BPSK.

\section{Split and Combing}

As we known, the length of the covert information $N$ basing on polar code equals $2^{n}$. Assuming covert communication is set on an 802.11a/g PHY layer. There are 48 subcarriers in a symbol in transmissions. The size of the covert information block and the carrier information block are incompatible, some all 0 or all 1 bits are filled in the first and the last symbol. As the length of code block becomes large, the symmetric capacity terms tend towards 0 or 1 . The length of the code block would be extended as much as possible. The number of filled bits $k$ can be expressed as

$$
k=\left(\left\lceil\frac{N}{48}\right\rceil+1\right) \cdot 48-N
$$

The symbol construction of subcarrier signal is illustrated in Figure 5. The blue code blocks are filled bits which can also be used to judge the beginning and end of the covert information code block. The yellow code blocks are covert information code block. Finite covert code block would be split into multiple blocks and combined with carrier information block. When we receive the subcarrier signal, subcarrier

Figure 5 Symbol construction of subcarrier signal

signal would be demodulated modulated to obtain the residual signal. Then, the residual signal would be demodulated to obtain covert code block. As is illustrated

Figure 6 Block extraction of covert information

in Figure 6, the covert information block would be extracted from the covert code block. Then, the covert information code block would be decoded by maximum likelihood decoding.

\section{Maximum Likelihood Decoding}

\section{A Successive Cancellation (SC) decoding}

Polar code is a Gn-coset code with parameter $\left(N, K, A, u_{A}\right)$ where $K$ is the code dimension and specifies the size of information set $A$. Covert message $u_{1}^{N}$ would be encoded into $x_{1}^{N}, x_{1}^{N}$ would be transmitted through the channel $W^{N}$ and Bob would receive $y_{1}^{N}$. Thus, Bob needs a corresponding decoder which could generate an accurate estimate $\hat{u}_{1}^{N}$ of $u_{1}^{N}$ with given knowledge of $A$, ua and $y_{1}^{N}$. Because parts of the codewords are frozen bits, the decoder can directly set $\hat{u}_{A c}=u_{A c}$. The task is just to estimate $\hat{u}_{A}$ of $u_{A}$. Arıkan has given a specific successive cancellation (SC) decoder. The SC decoder can generate its estimate of $u_{A}$ with given parameter 
$\left(N, K, A, u_{A}\right)$ by computing

$$
\hat{u}_{i} \triangleq\left\{\begin{array}{l}
u_{A c} \quad \text { if } i \in A c \\
h_{i}\left(y_{1}^{N}, \hat{u}_{1}^{i-1}\right) \quad \text { if } i \in A
\end{array}\right.
$$

where $i \in[1, N]$ and the decision functions are defined as

$$
h_{i}\left(y_{1}^{N}, \hat{u}_{1}^{i-1}\right) \triangleq \begin{cases}0, & \text { if } L_{N}^{(i)}\left(y_{1}^{N}, \hat{u}_{1}^{i-1}\right) \geq 0 \\ 1, & \text { if } L_{N}^{(i)}\left(y_{1}^{N}, \hat{u}_{1}^{i-1}\right)<0\end{cases}
$$

and the Log-Likelihood Ratio (LLR) can be expressed as

$$
L_{N}^{(i)}\left(y_{1}^{N}, \hat{u}_{1}^{i-1}\right) \triangleq \ln \left(\frac{W_{N}^{(i)}\left(y_{1}^{N}, \hat{u}_{1}^{i-1} \mid 0\right)}{W_{N}^{(i)}\left(y_{1}^{N}, \hat{u}_{1}^{i-1} \mid 1\right)}\right)
$$

To calculate the LLR, we can use function $f$ and function $g$

$$
\left\{\begin{array}{l}
f(a, b) \triangleq \ln \left(\frac{1+e^{a+b}}{e^{a}+e^{b}}\right) \\
g\left(a, b, u_{s}\right) \triangleq(-1)^{u_{s}} a+b
\end{array}\right.
$$

We can equivalently express the LLR as

$$
\left\{\begin{array}{c}
L_{N}^{(2 i-1)}\left(y_{1}^{N}, \hat{u}_{1}^{2 i-2}\right)=f\left(L_{N / 2}^{(i)}\left(y_{1}^{N / 2}, \hat{u}_{1, o}^{2 i-2} \oplus \hat{u}_{1, e}^{2 i-2}\right), L_{N / 2}^{(i)}\left(y_{N / 2+1}^{N}, \hat{u}_{1, e}^{2 i-2}\right)\right) \\
L_{N}^{(2 i)}\left(y_{1}^{N}, \hat{u}_{1}^{2 i-1}\right)=g\left(L_{N / 2}^{(i)}\left(y_{1}^{N / 2}, \hat{u}_{1, o}^{2 i-2} \oplus \hat{u}_{1, e}^{2 i-2}\right), L_{N / 2}^{(i)}\left(y_{N / 2+1}^{N}, \hat{u}_{1, e}^{2 i-2}\right), \hat{u}_{2 i-1}\right)
\end{array}\right.
$$

Thus, the task of calculating an LLR at length $N$ is converted to calculating two LLRs at length $N / 2$. This recursion can be continued down until the block length is 1, at which point the LLRs can be computed directly. To estimate the complexity of LLR calculations, we denote the Kolmogorov complexity of decoding over $k \in$ $[N, N / 2, N / 4, \cdots, 1]$ as $K_{L}(k)$. We have the complexity bound

$$
K_{L}(k) \leq 2 K_{L}(k / 2)+c_{A}
$$

where $c_{A}$ is the worst case complexity of combining two LLRs at length $k / 2$ into an LLR at length $k$. We can obtain the bound

$$
K_{L}(N) \leq N K_{L}(1)+c_{A}=\mathrm{O}(N)
$$

We can obtain the overall decoder complexity

$$
K_{D}(N) \leq K K_{L}(N) \leq N K_{L}(N)=\mathrm{O}\left(N^{2}\right)
$$

This overall complexity corresponds to a decoder whose DEs work privately. With sharing any partial results between DEs, the decoder could have a more efficient implementation with overall complexity $\mathrm{O}(N \log N)$. 


\section{A Successive Cancellation List (SCL) decoding}

Even the code block length are comparable, the performance of polar codes is inferior to LDPC and turbo codes. To improve the performance of polar code, Ido [24] have proposed a successive-cancellation list (SCL) decoder. In SCL decoder, $L$ decoding paths are considered concurrently at each decoding stage. By comparing the path metric (PM) of $L$ results, the most likely estimate is selected as the decoder output.

The path metric $(\mathrm{PM})$ of path $l(l \in[1,2 \cdots, L])$ can be expressed as

$$
P M_{l}^{(i)} \triangleq \sum_{j=1}^{i} \ln \left(1+\exp \left(-\left(1-2 \hat{u}_{j}[l]\right) \cdot L_{N}^{(j)}\right)\right)
$$

where $L_{N}^{(i)}=\ln \left(\frac{W_{N}^{(i)}\left(y_{1}^{N}, \hat{u}_{1}^{i-1} \mid 0\right)}{W_{N}^{(i)}\left(y_{1}^{N}, \hat{u}_{1}^{i-1} \mid 1\right)}\right)$. For any two paths $l_{1}, l_{2} \in[1,2 \cdots, L]$, when we have $P M_{l_{1}}^{(i)}>P M_{l_{2}}^{(i)}$, we can equivalently obtain

$$
W_{N}^{(i)}\left(y_{1}^{N}, \hat{u}_{1}^{i-1}\left[l_{1}\right] \mid \hat{u}_{1}^{i}\left[l_{1}\right]\right)<W_{N}^{(i)}\left(y_{1}^{N}, \hat{u}_{1}^{i-1}\left[l_{2}\right] \mid \hat{u}_{1}^{i}\left[l_{2}\right]\right)
$$

We can obtain that the greater transition probability $W_{N}^{(i)}$, the smaller the path metric $P M_{l}^{(i)}$. The Eq.38 can be expressed as

$$
P M_{l}^{(i)} \approx\left\{\begin{array}{c}
P M_{l}^{(i-1)}, \quad \text { if } \hat{u}_{i}[l]=\delta\left(L_{N}^{(i)}[l]\right) \\
P M_{l}^{(i-1)}+\left|L_{N}^{(i)}[l]\right|, \quad \text { if } \hat{u}_{i}[l] \neq \delta\left(L_{N}^{(i)}[l]\right)
\end{array}\right.
$$

where $\delta(x)=\frac{1}{2}(1-\operatorname{sign}(x))$ and $P M_{l}^{(0)}=0$. Assuming the decoding path $L=4$, the decoding process can be shown in Figure 7 . Let gray paths as the abandoned paths, black paths as adopted paths.

Figure 7 Decoding process of SCL decoder with $L=4$

\section{Results and discussion}

In this section, the undetectability and reliability of the proposed scheme are discussed by simulation results. The dedicated radio-frequency source is set on an 802.11a/g PHY layer. There are 48 subcarriers in a symbol. The wireless covert channel is performed on 1000000 code blocks. Simulation experiments are carried out in wireless channel models of TGn channel models [25]. The list number $L$ of SCL decoder is 16 . In some simulations, covert information with $\mathrm{BCH}$ code is chosen for comparison.

\section{Reliability}

The reliability is measured by BLER. The length of the BCH code block is 1023 bits, and the information bits are 513 bits. The length of the polar code block is $1024 \mathrm{bits}$, and the information bits are 512 bits. We let the power ratio of subcarrier signal and AWGN as $S N R$, the power ratio of carrier signal and secret signal as 
CSR. After 100 cycles of simulation, the average BLER of wireless covert communication with BCH code block is shown in Figure 8.

The BLER of wireless covert channel with polarized dirty constellation is shown

Figure 8 BLER of wireless covert communication with $\mathrm{BCH}$ codes

in Figure 9,10. In Figure 9, the received polar code blocks are decoded with SC decoder. To improve the reliability of covert communication, we decode the same polar code blocks with SCL decoder. The number of decoding paths is 16. And the BLER of SCL decoder is shown in Figure 10.

As shown in Figures 8,9 and 10, when the $S N R$ of subcarrier signal is $15 \mathrm{~dB}$, covert

Figure 9 BLER of polarization dirty constellation with SC decoder

Figure 10 BLER of polarization dirty constellation with SCL decoder

communication with $\mathrm{BCH}$ code can obtain BLER as $1 \mathrm{e}-6$ at $C S R=12 \mathrm{~dB}$. And WCC-PDC can obtain the same BLER at $15 \mathrm{~dB}$ with SC decoder. When we decode the covert information with SCL decoder, we can obtain BLER as 1e-6 at $C S R=16$ $\mathrm{dB}$. Lower $C S R$ means that we can transmit much more covert information reliably, with the same transmission power. Thus, wireless covert communication with polarized dirty constellation can obtain better reliability than covert communication with $\mathrm{BCH}$ code.

\section{Undetectability}

Undetectability is also an important measure of wireless covert communication. The undetectability is inspected by 'KL divergence' and 'KS distance'. The undetectability measures of $I$ vectors, $Q$ vectors, magnitudes, and phase are detected with constellation errors. The K-L divergence and KS-distance are detected in the range of $S N R=15, \ldots 30 \mathrm{~dB}$ and $C S R=10, \ldots 30 \mathrm{~dB}$. To measure the undetectability of different code blocks, we mark the points of $\mathrm{KL}$ divergence at $\mathrm{BLER}=1 \mathrm{e}-5$. We let the red point as wireless covert communication with $\mathrm{BCH}$ code. Let the polar code with SC decoder and SCL decoder as green point and yellow point. The K-L divergence of $I$ vectors, $Q$ vectors, magnitudes and phase are shown in Figures 11, 12,13 , and 14 .

As shown in Figures 11,12,13 and 14, with the same BLER as 1e-5, wireless covert

Figure $11 \mathrm{KL}$ divergence of I vectors in constellation errors

Figure $12 \mathrm{KL}$ divergence of $\mathrm{Q}$ vectors in constellation errors 
Figure $13 \mathrm{KL}$ divergence of magnitudes in constellation errors

Figure $14 \mathrm{KL}$ divergence of phase in constellation errors

communication with polarized dirty constellation can obtain lower KL-divergence than covert communication with BCH code. And polar code with SCL decoder can obtain the best undetectability. Additionally, the KL divergence nearly no longer changes at the position of the yellow point. Because of the presence of AWGN, the detector cannot set the threshold of KL divergence at a very small number, which may increase the probability of false alarm.

The KS distance of wireless covert communication is shown in Figures 15,16,17

Figure $15 \mathrm{KS}$ distance of I vectors in constellation errors

Figure $16 \mathrm{KS}$ distance of $\mathrm{Q}$ vectors in constellation errors

Figure $17 \mathrm{KS}$ distance of magnitudes in constellation errors

Figure $18 \mathrm{KS}$ distance of phase in constellation errors

and 18. As the figures show, with the same BLER, wireless covert communication with polarized dirty constellation can obtain lower $\mathrm{KS}$ distance than $\mathrm{BCH}$ code. Therefore, it can be obtained that the undetectability of covert communication improves significantly with polar codes. Additionally, better undetectability can be obtained with SCL decoder.

\section{Conclusion}

In this paper, we proposed a wireless covert channel with polarization dirty constellation in backscatter communication system. We extended the polarization theory to wireless covert communication. Furthermore, it was proved that part of covert information can be transmitted with Shannon capacity basing on polar codes when the length of code block is long enough. We polarized the covert channel in wireless covert communication and decoded the covert messages with SC decoder and SCL decoder. It was proved that we can obtain better reliability and undetectability with polarization dirty constellation.

Although the reliability and undetectability can be improved significantly with polar codes, its performance is limited by the length of code block and decoding method. The polarization of the code block will be incomplete when the length of covert code block is not long enough. Thus, it is necessary to find a better way to combine polar code and covert communication in our future work. 
Acknowledgements

This work was supported by the National Natural Science Foundation of China.

Funding

This work was supported by the National Natural Science Foundation of China (Grants No. U1836104, 61772281, 61702235, 61801073, 61931004, 62072250) ,Universities Natural Science Research Project of Jiangsu Province (No. 20KJB510037), open research fund of National Mobile Communications Research Laboratory, Southeast University (No.2021D11), the Startup Foundation for Introducing Talent of NUIST.

Abbreviations

AWGN: Additive white gaussian noise

SNR: Signal noise ratio

OFDM: Orthogonal frequency division multiplexing

BLER: Block error ratio

BER: Bit error ratio

$\mathrm{BCH}$ code: Bose-Chaudhuri-Hocquenghem codes

WCC-DC: Wireless covert communication with dirty constellation

LLR: Log-Likelihood Ratio

Availability of data and materials

Not available online. Please contact corresponding author for data requests.

Competing interests

The authors declare that they have no competing interests.

Authors' contributions

SQ, GJL, and JFS conceived the study. SQ and GJL designed and modeled. SQ, XPJ, and WWL analyzed the performance. SQ, GJL, and XPJ interpreted the results and wrote the manuscript. All authors read and approved the final manuscript.

\section{Authors' information}

Sen Qiao received the M.S. degree in control engineering from Jiangsu University of Science and Technology, Zhenjiang, China, in 2017. He is currently pursuing the PhD degree in Nanjing University of Information Science and Technology. His research interests include wireless communications and covert communications.

GuangJie Liu received the B.S. degree in information engineering and the Ph.D. degree in control science and engineering from the Nanjing University of Science and Technology, Nanjing, in 2002 and 2007, respectively. From 2016 to 2017, he was a Visiting Scholar with the Department of Computer Science, University of California at Davis, Davis, CA, USA. He is currently an Professor with the Nanjing University of Information Science and Technology. His research interests are network and communication security.

Jianfeng Shi received the B.S. degree in communication engineering from Nanjing Normal University, Nanjing, China, in 2014, and the Ph.D. degree from the National Mobile Communication Research Laboratory, Southeast University, in 2019. He is currently a Lecture with the School of Electronic and Information Engineering, Nanjing University of Information Science and Technology, Nanjing. From September 2017 to September 2018, he was a visiting student with the Department of Electrical Computer Engineering, McGill University, Canada. His research interests include integrated satellite and terrestrial networks, user-centric networks, and dynamic optimization. He served as a TPC Member for IEEE ICC, WCNC, and CIC ICCC from 2015 to 2018, and 2020.

XiaoPeng Ji received the BS degree in electrical and computer engineering and the MS and PhD degrees in control science and engineering from the Nanjing University of Science and Technology, Nanjing, in 2007, 2009, and 2013, respectively. He is currently an Associate Professor with the Nanjing University of Information Science and Technology. His research interests include multimedia communication and wireless sensor networks.

WeiWei Liu received the B.S. degree in automation and the Ph.D. degree in control science and engineering from the Nanjing University of Science and Technology, Nanjing, in 2010 and 2015, respectively. From 2014 to 2015, he was a Visiting Scholar with the Department of Computer Science, University of California at Davis, Davis, CA, USA $\mathrm{He}$ is currently an Associate Professor with the School of Automation, Nanjing University of Science and Technology. His research interests include multimedia signal processing and network traffic analysis. He has published over 30 articles in these areas, including the IEEE TRANSACTIONS ON INFORMATION FORENSICS AND SECURITY and Annals of Telecommunications. He is an active Reviewer of several journals, including Digital Signal Processing and Security and Communication Networks.

Author details

${ }^{1}$ School of Electrical and Information Engineering, Nanjing University of Information Science and Technology, Nanjing, China. ${ }^{2}$ National Mobile Communications Research Laboratory, Southeast University, Nanjing, China. ${ }^{3}$ School of Automation, Nanjing University of Science and Technology, Nanjing, China.

References

1. Bi, S., Ho, C.K., Zhang, R.: Wireless powered communication: Opportunities and challenges. IEEE Communications Magazine 53(4), 117-125 (2015)

2. Bi, S., Zeng, Y., Zhang, R.: Wireless powered communication networks: An overview. IEEE Wireless Communications 23(2), 10-18 (2016)

3. Zhao, W., Wang, G., Atapattu, S., Tsiftsis, T.A., Ma, X.: Performance analysis of large intelligent surface aided backscatter communication systems. IEEE Wireless Communications Letters 9(7), 962-966 (2020)

4. Liu, V., Parks, A., Talla, V., Gollakota, S., Wetherall, D., Smith, J.R.: Ambient backscatter: Wireless communication out of thin air. ACM SIGCOMM Computer Communication Review 43(4), 39-50 (2013) 
5. Yang, G., Liang, Y.-C., Zhang, R., Pei, Y.: Modulation in the air: Backscatter communication over ambient ofdm carrier. IEEE Transactions on Communications 66(3), 1219-1233 (2017)

6. Dutta, A., Saha, D., Grunwald, D., Sicker, D.: Secret agent radio: Covert communication through dirty constellations. In: International Workshop on Information Hiding, pp. 160-175 (2012). Springer

7. Cek, M.E., Savaci, F.: Stable non-gaussian noise parameter modulation in digital communication. Electronics letters 45(24), 1256-1257 (2009)

8. Cek, M.: Covert communication using skewed $\alpha$-stable distributions. Electronics Letters 51(1), 116-118 (2015)

9. Kitano, T., Iwai, H., Sasaoka, H.: A wireless steganography technique by embedding ds-ss signal in digital mobile communication systems. Science Engineering Review of Doshisha University 52, 127-134 (2011)

10. Ma, H., Yi, X., Wu, X., Gu, D., Pan, H., Zheng, C.: A capacity self-adaption information hiding algorithm based on rs code. In: 2014 International Conference on Multisensor Fusion and Information Integration for Intelligent Systems (MFI), pp. 1-8 (2014). IEEE

11. Kellogg, B., Parks, A., Gollakota, S., Smith, J.R., Wetherall, D.: Wi-fi backscatter: Internet connectivity for rf-powered devices. In: Proceedings of the 2014 ACM Conference on SIGCOMM, pp. 607-618 (2014)

12. Qian, J., Gao, F., Wang, G., Jin, S., Zhu, H.: Noncoherent detections for ambient backscatter system. IEEE Transactions on Wireless Communications 16(3), 1412-1422 (2016)

13. Wang, G., Gao, F., Fan, R., Tellambura, C.: Ambient backscatter communication systems: Detection and performance analysis. IEEE Transactions on Communications 64(11), 4836-4846 (2016)

14. Shahzad, K., Zhou, X.: Covert communication in backscatter radio. In: ICC 2019-2019 IEEE International Conference on Communications (ICC), pp. 1-6 (2019). IEEE

15. Yan, S., Cong, Y., Hanly, S.V., Zhou, X.: Gaussian signalling for covert communications. IEEE Transactions on Wireless Communications 18(7), 3542-3553 (2019)

16. Wang, Y., Yan, S., Yang, W., Huang, Y., Liu, C.: Energy-efficient covert communications for bistatic backscatter systems. IEEE Transactions on Vehicular Technology 70(3), 2906-2911 (2021)

17. Cao, P., Liu, W., Liu, G., Ji, X., Zhai, J., Dai, Y.: A wireless covert channel based on constellation shaping modulation. Security and Communication Networks 2018 (2018)

18. Simmons, G.J.: The prisoners' problem and the subliminal channel. In: Advances in Cryptology, pp. 51-67 (1984). Springer

19. Cover, T.M.: Elements of Information Theory. John Wiley \& Sons, New Jersey (1999)

20. Lehmann, E.: Testing statistical hypotheses: the story of a book. In: Selected Works of EL Lehmann, pp. 995-999. Springer, Boston (2012)

21. Lee, S., Baxley, R.J., McMahon, J.B., Frazier, R.S.: Achieving positive rate with undetectable communication over mimo rayleigh channels. In: 2014 IEEE 8th Sensor Array and Multichannel Signal Processing Workshop (SAM), pp. 257-260 (2014). IEEE

22. Arikan, E.: Channel polarization: A method for constructing capacity-achieving codes for symmetric binary-input memoryless channels. IEEE Transactions on information Theory 55(7), 3051-3073 (2009)

23. Gallager, R.G.: Information Theory and Reliable Communication vol. 2. Springer, New York (1968)

24. Tal, I., Vardy, A.: List decoding of polar codes. IEEE Transactions on Information Theory 61(5), 2213-2226 (2015)

25. Group, I...W., et al.: Part 11: wireless lan medium access control (mac) and physical layer (phy) specifications: higher-speed physical layer extension in the 2.4 ghz band. ANSI/IEEE Std 802.11 (1999) 
Figures

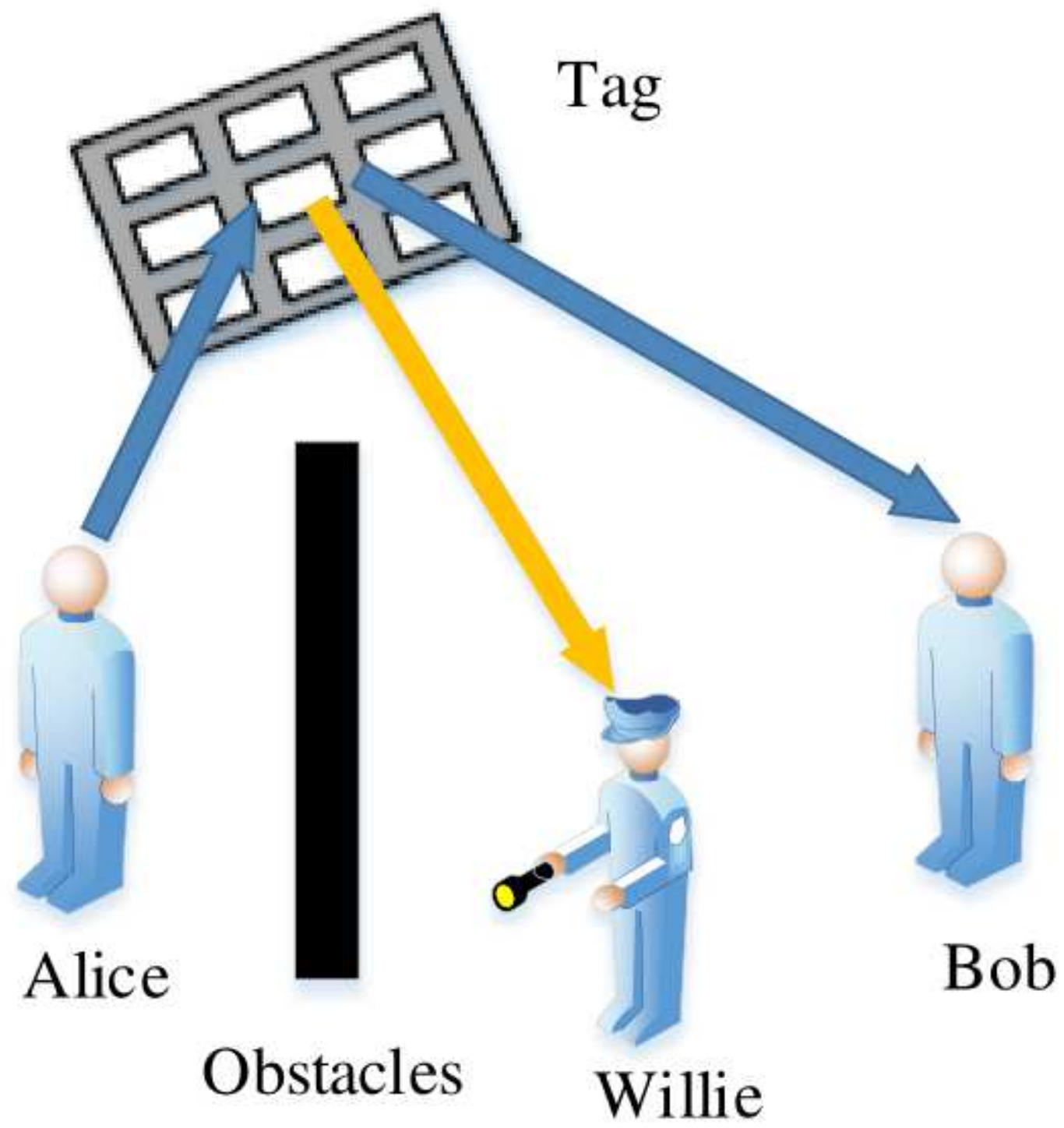

Figure 1

The framework of wireless covert communication 


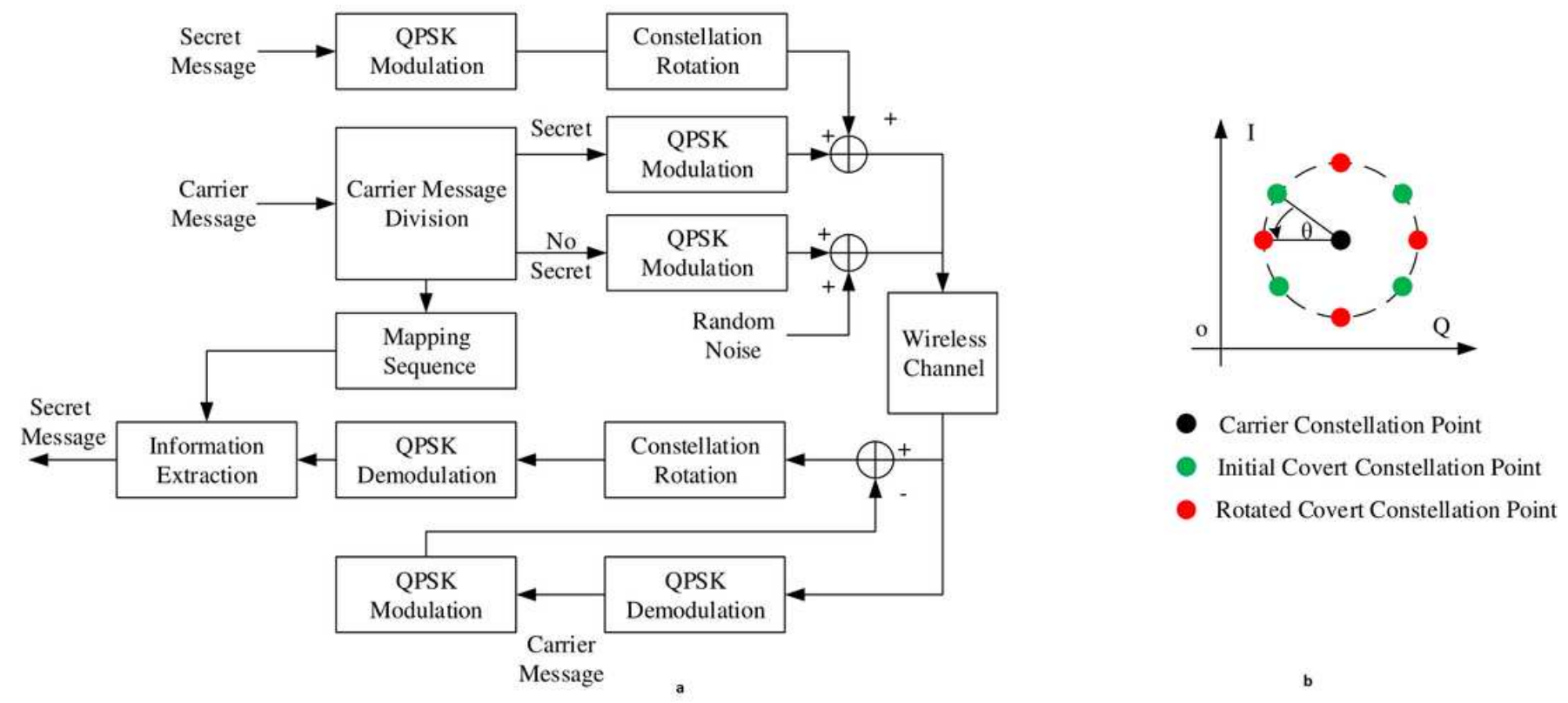

Figure 2

(a)The framework of wireless covert communication with dirty constellation(b)Rotation of Covert Constellation in WCC-DC

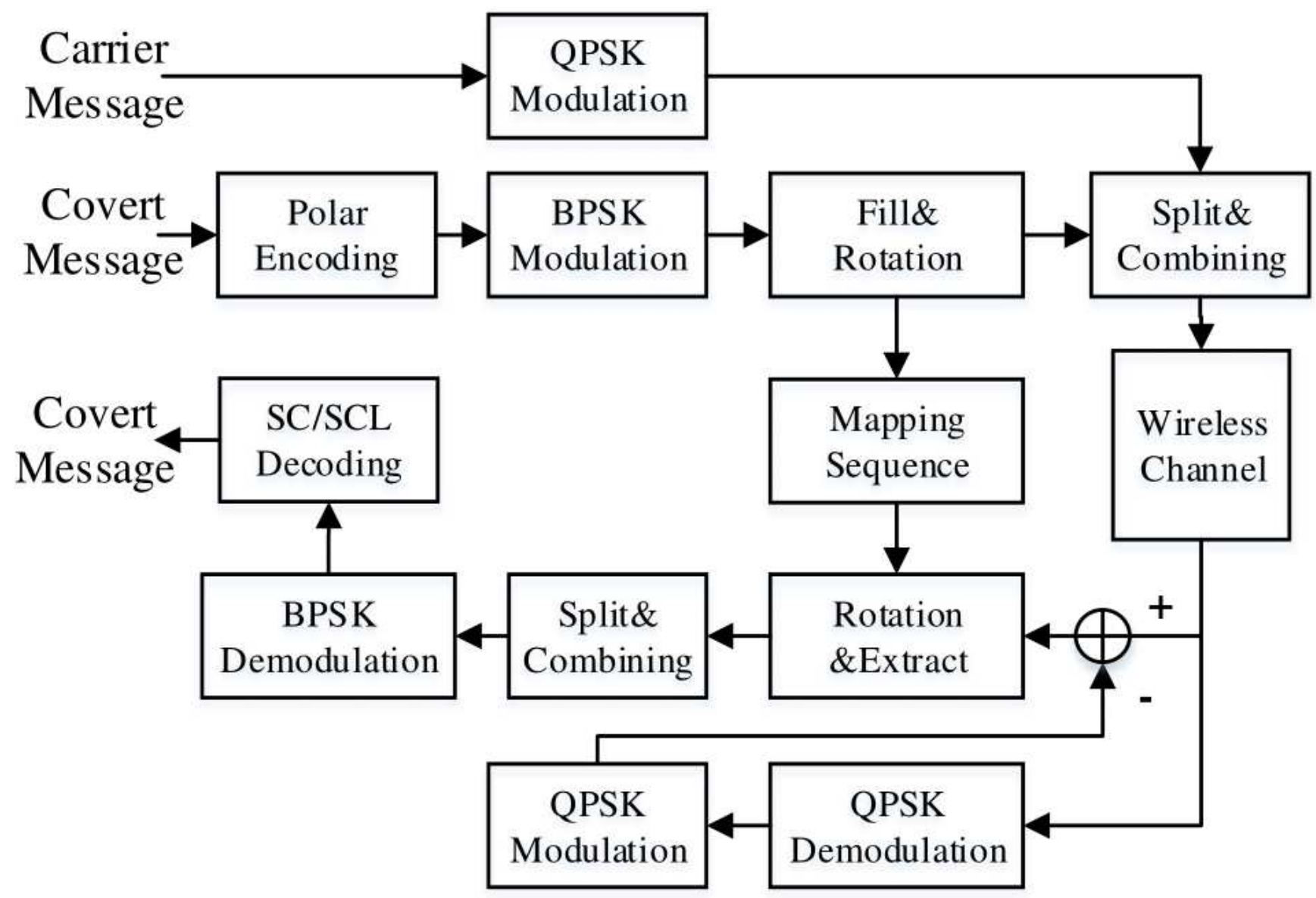


Figure 3

The schematic diagram of wireless covert communication with polarization dirty constellation

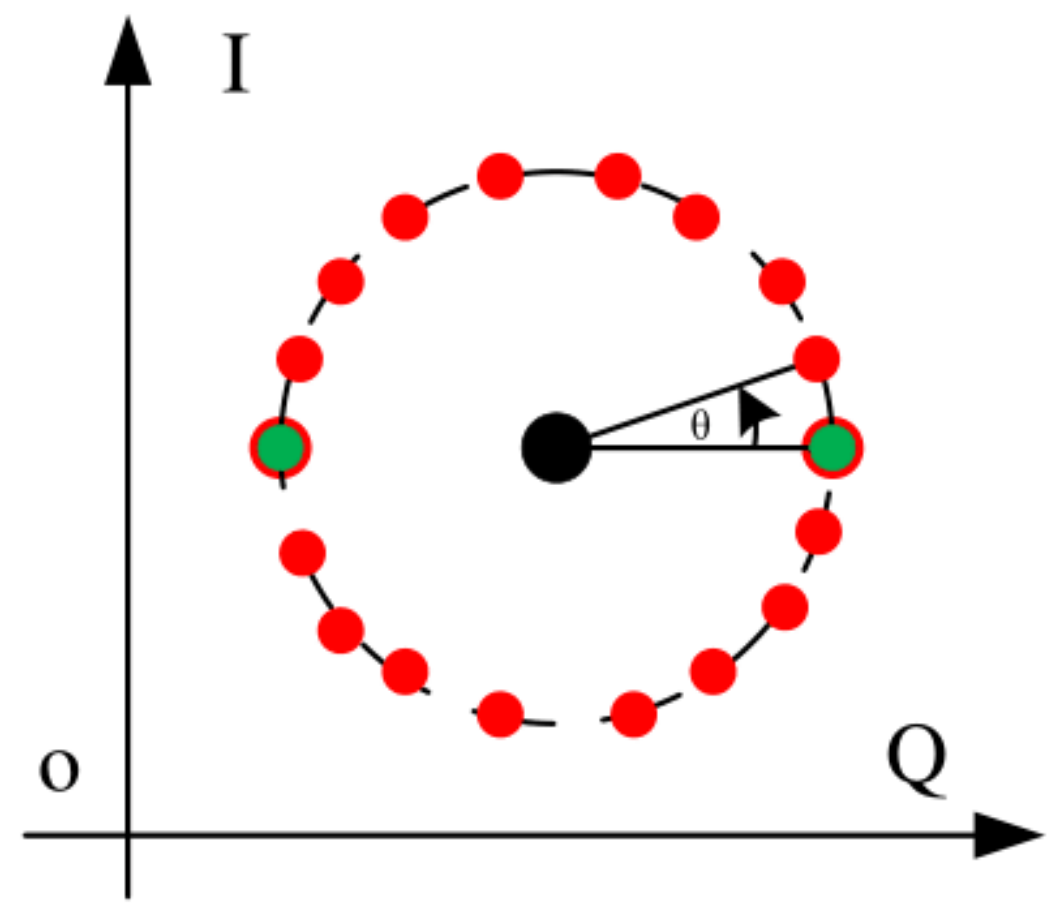

- Carrier Constellation Point Initial Covert Constellation Point Rotated Covert Constellation Point

Figure 4

Rotation of Polarized Covert Constellation 


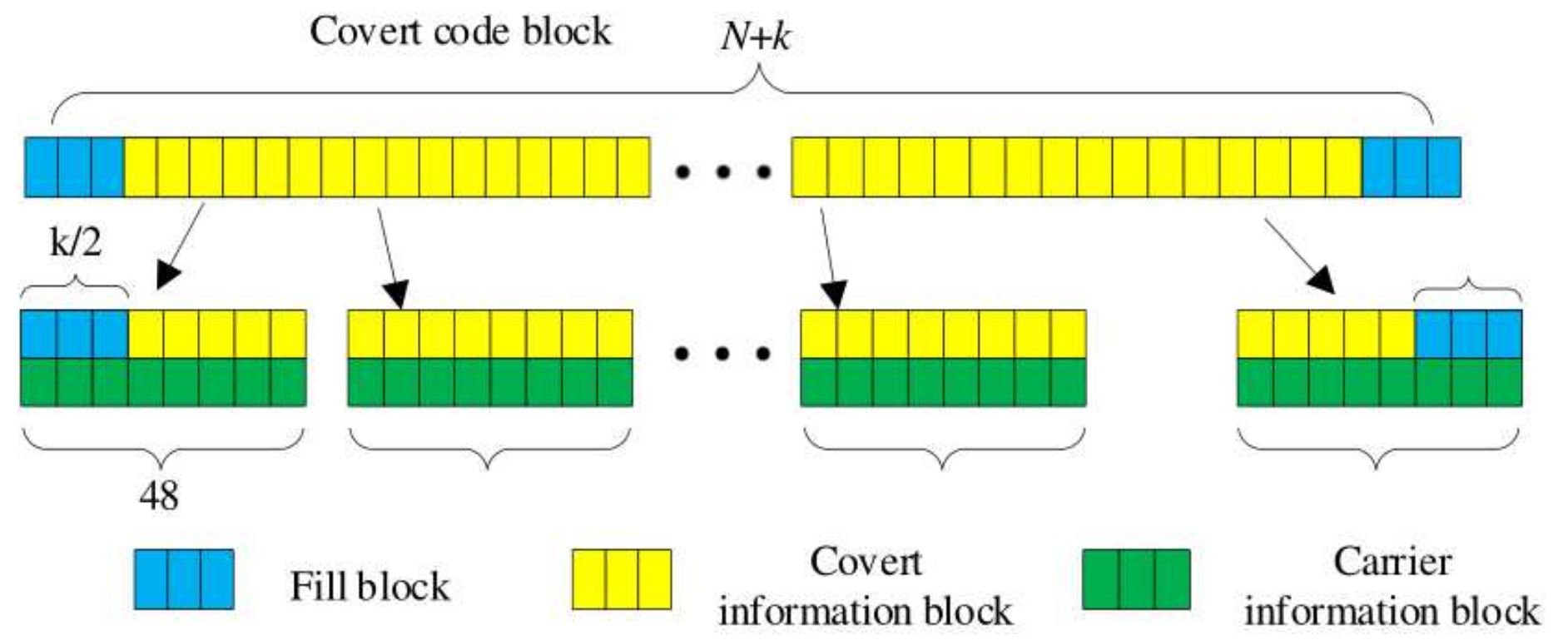

Figure 5

Symbol construction of subcarrier signal

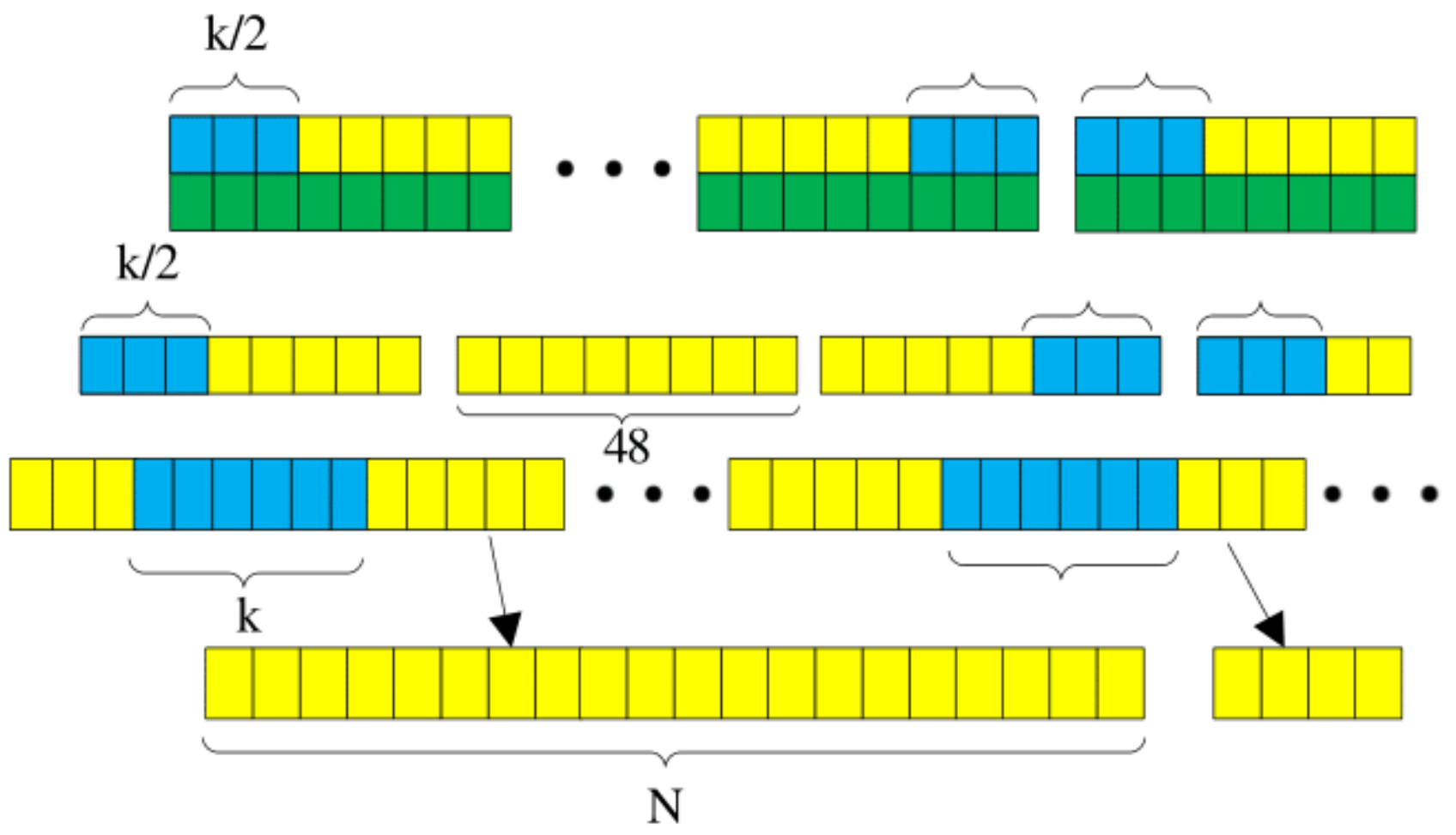

Figure 6

Block extraction of covert information 


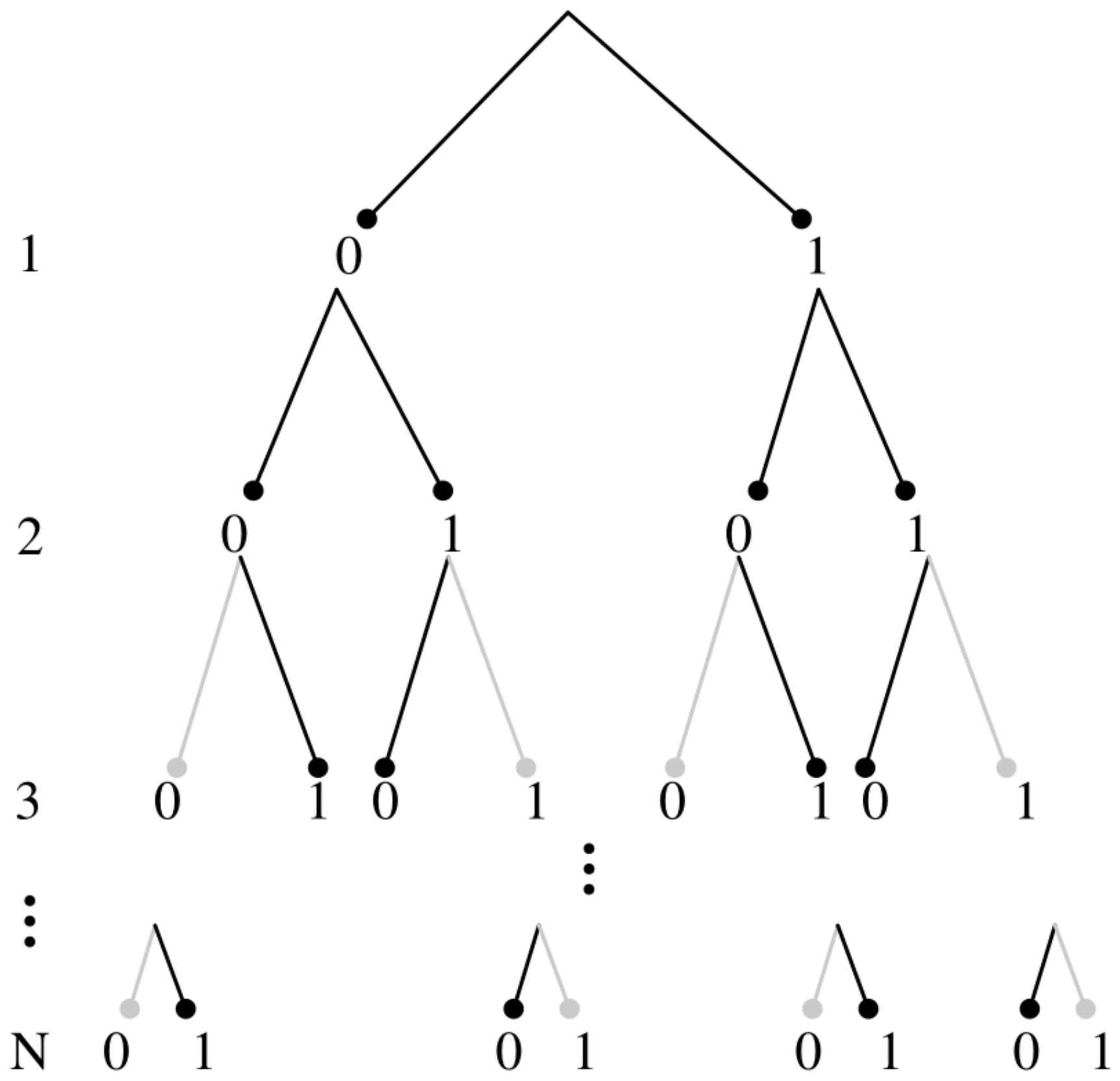

Figure 7

Decoding process of $\mathrm{SCL}$ decoder with $\mathrm{L}=4$ 


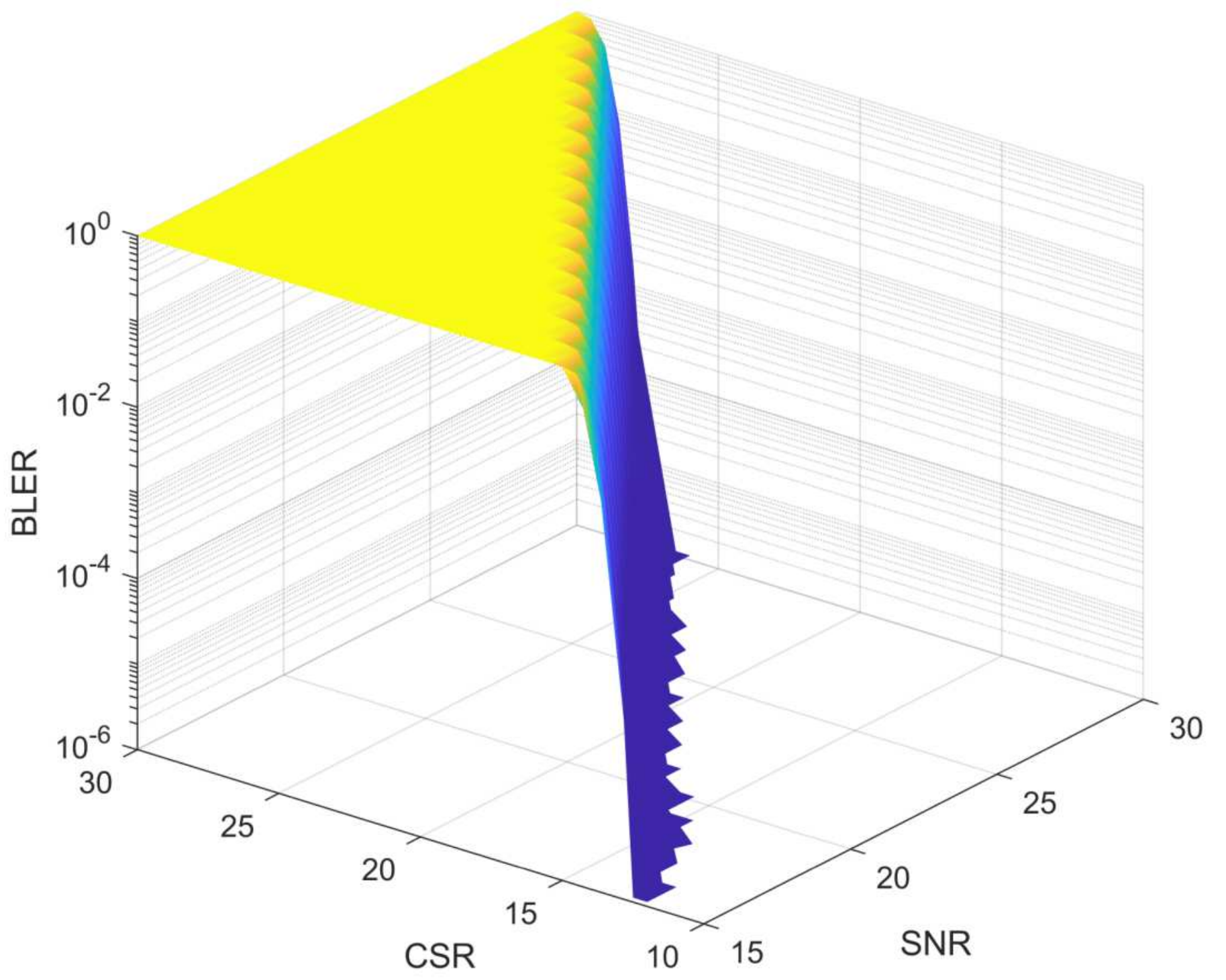

Figure 8

BLER of wireless covert communication with $\mathrm{BCH}$ codes 


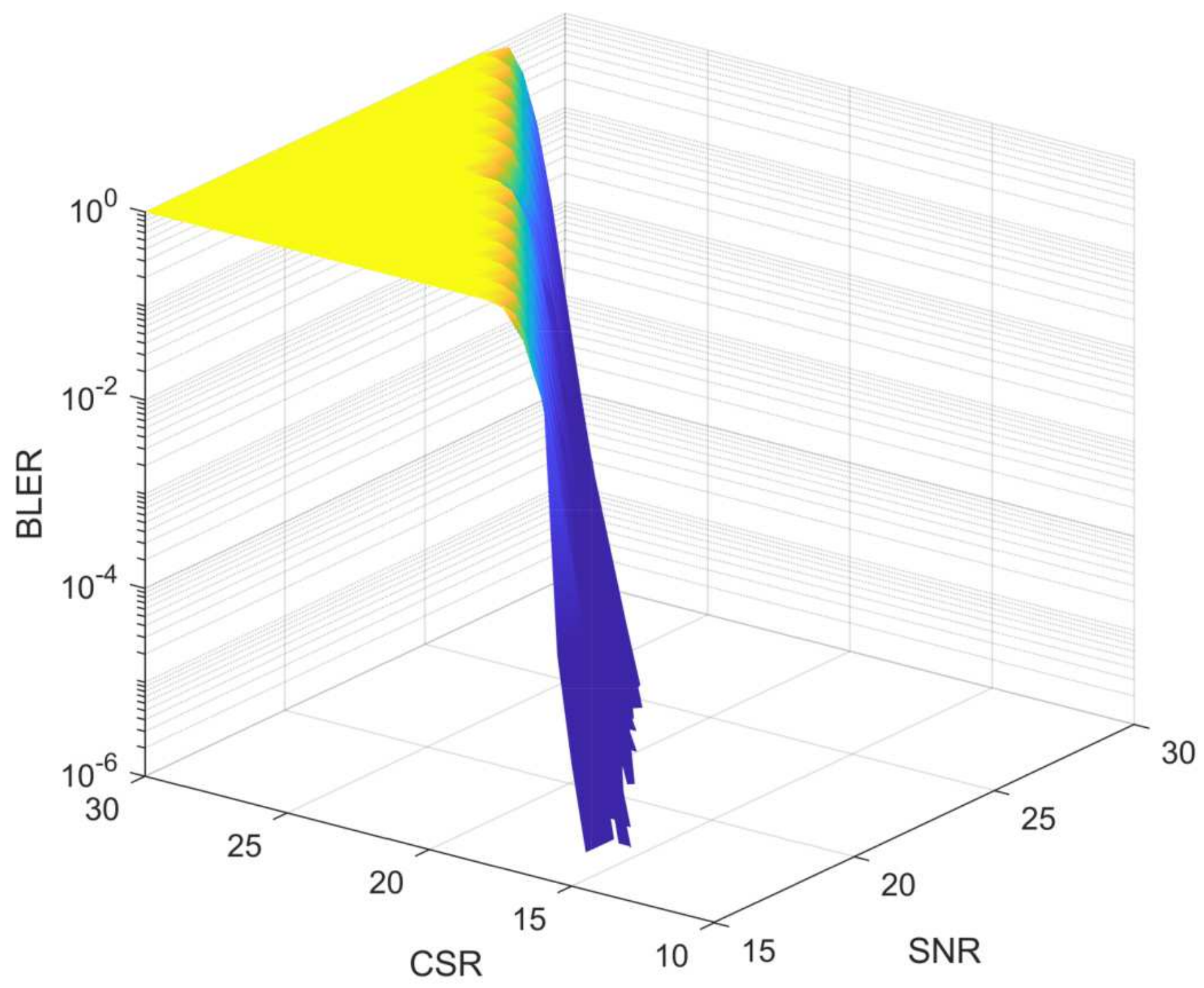

Figure 9

BLER of polarization dirty constellation with SC decoder 


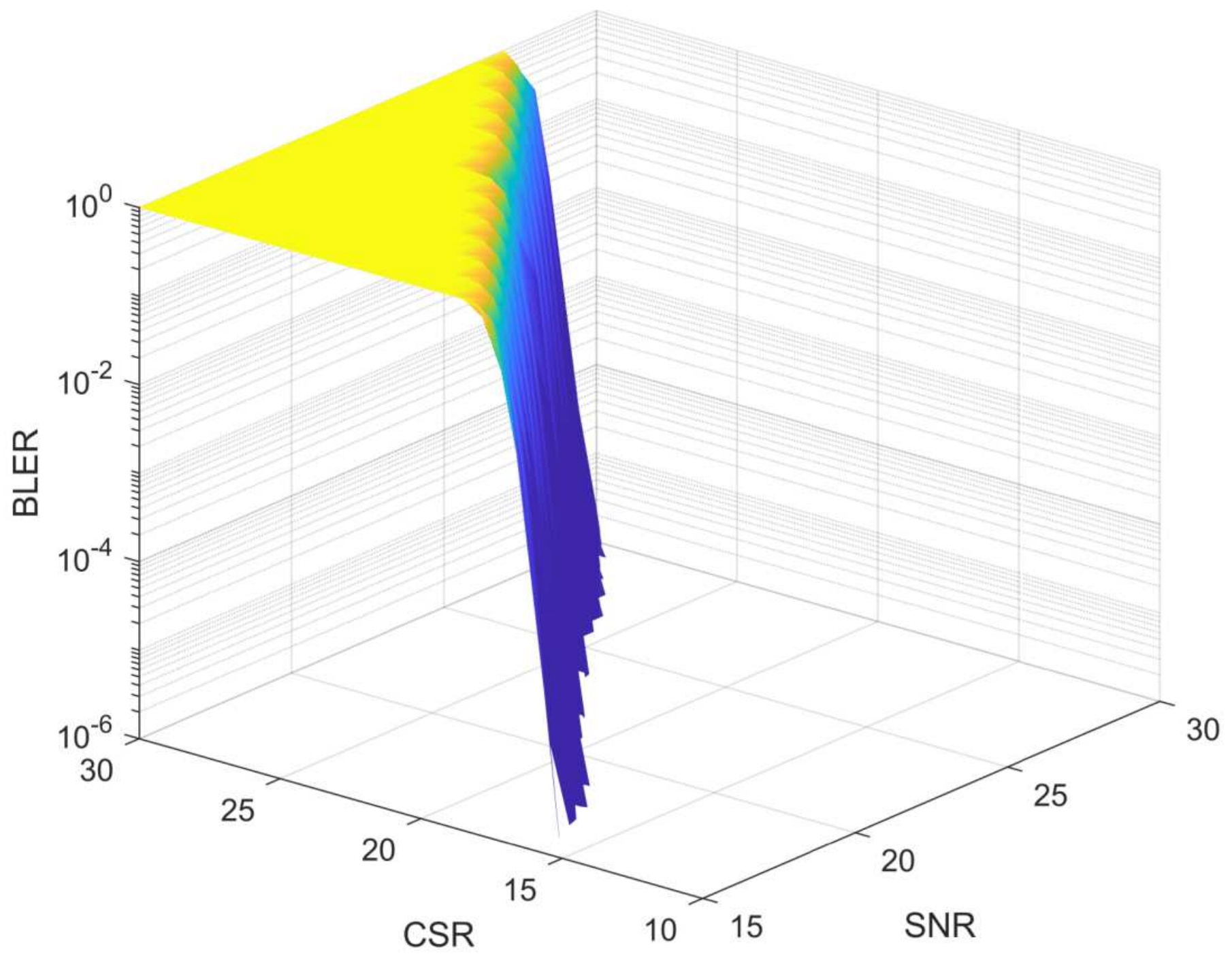

Figure 10

BLER of polarization dirty constellation with SCL decoder 


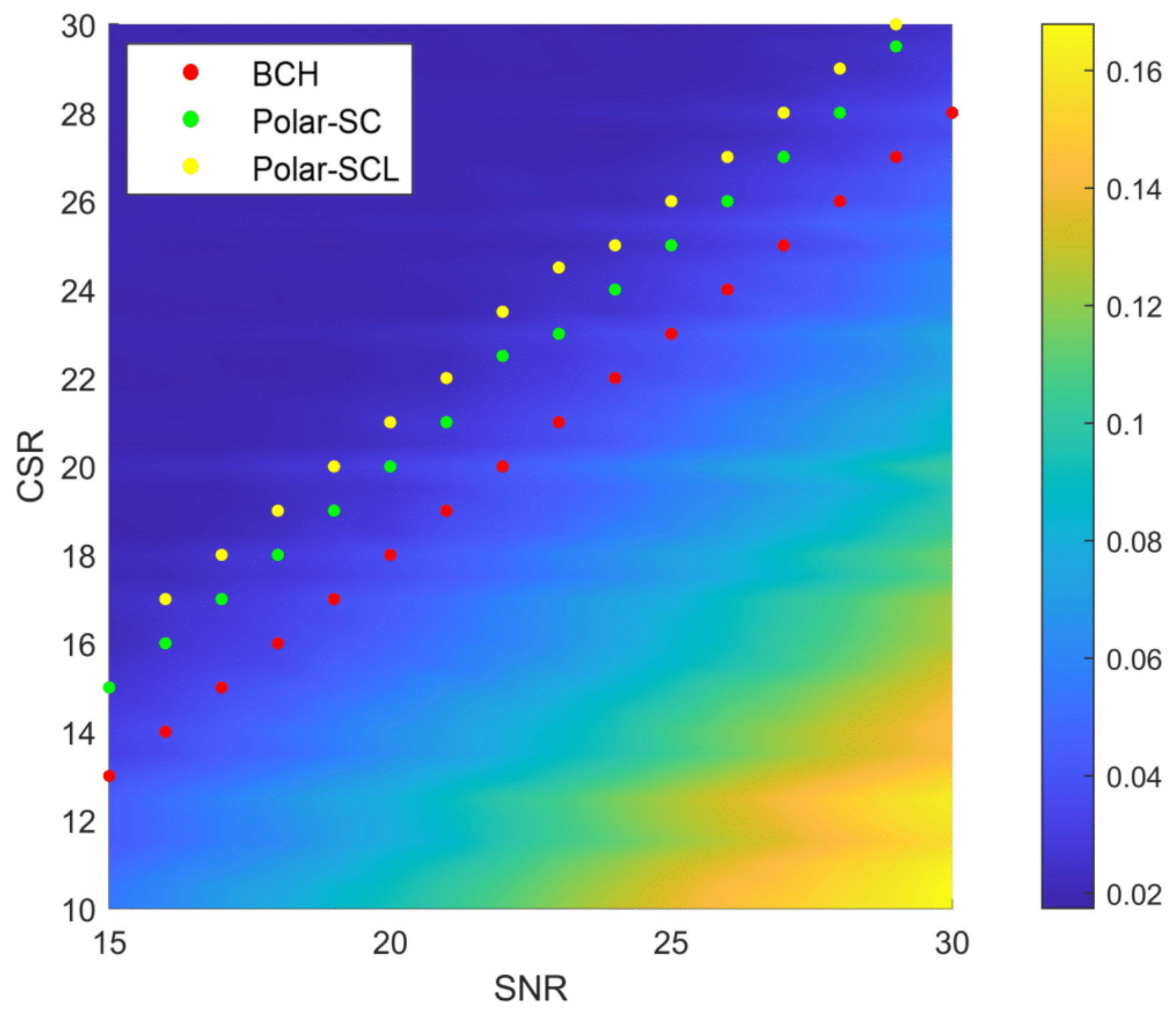

Figure 11

$\mathrm{KL}$ divergence of I vectors in constellation errors 


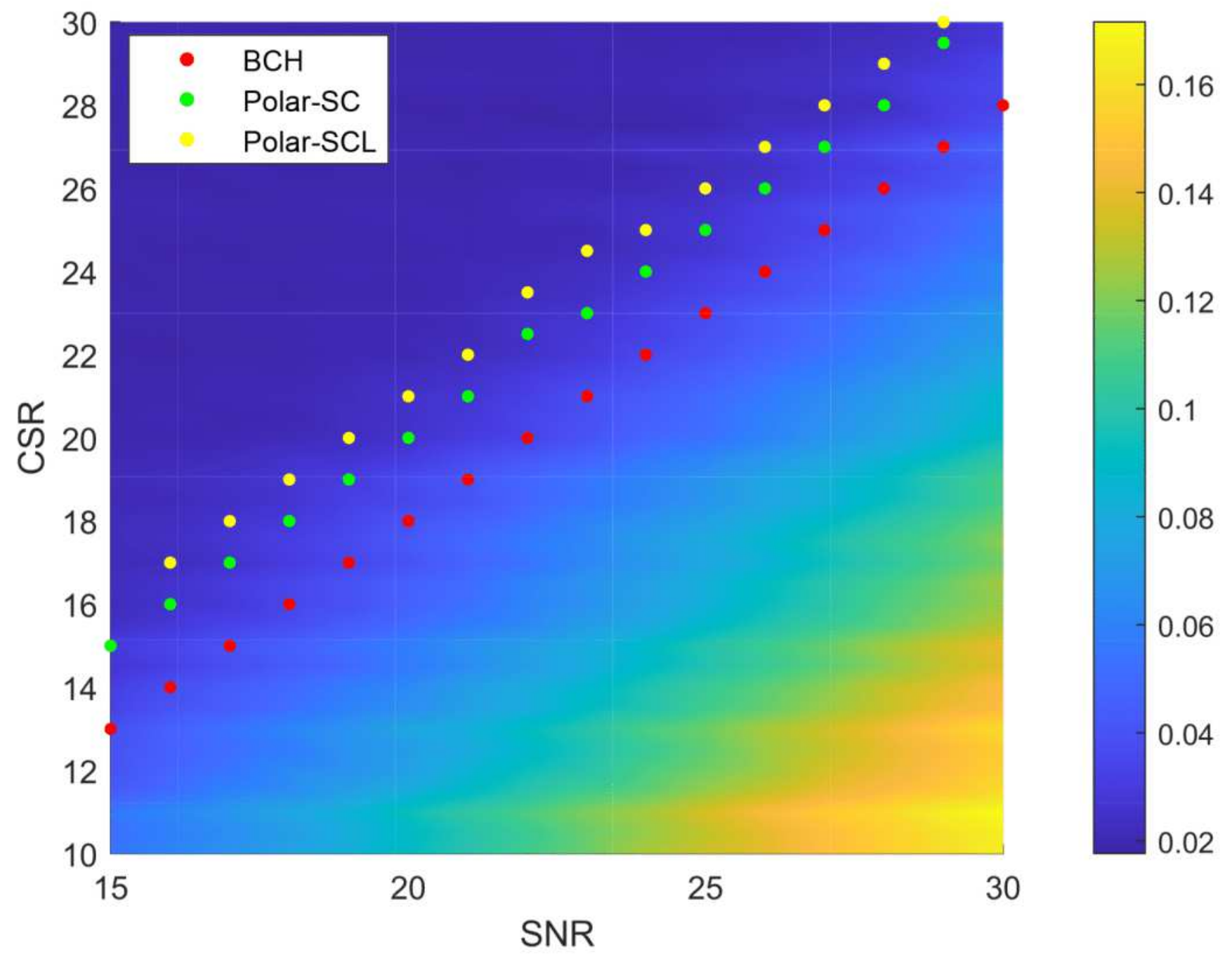

Figure 12

$\mathrm{KL}$ divergence of $\mathrm{Q}$ vectors in constellation errors 


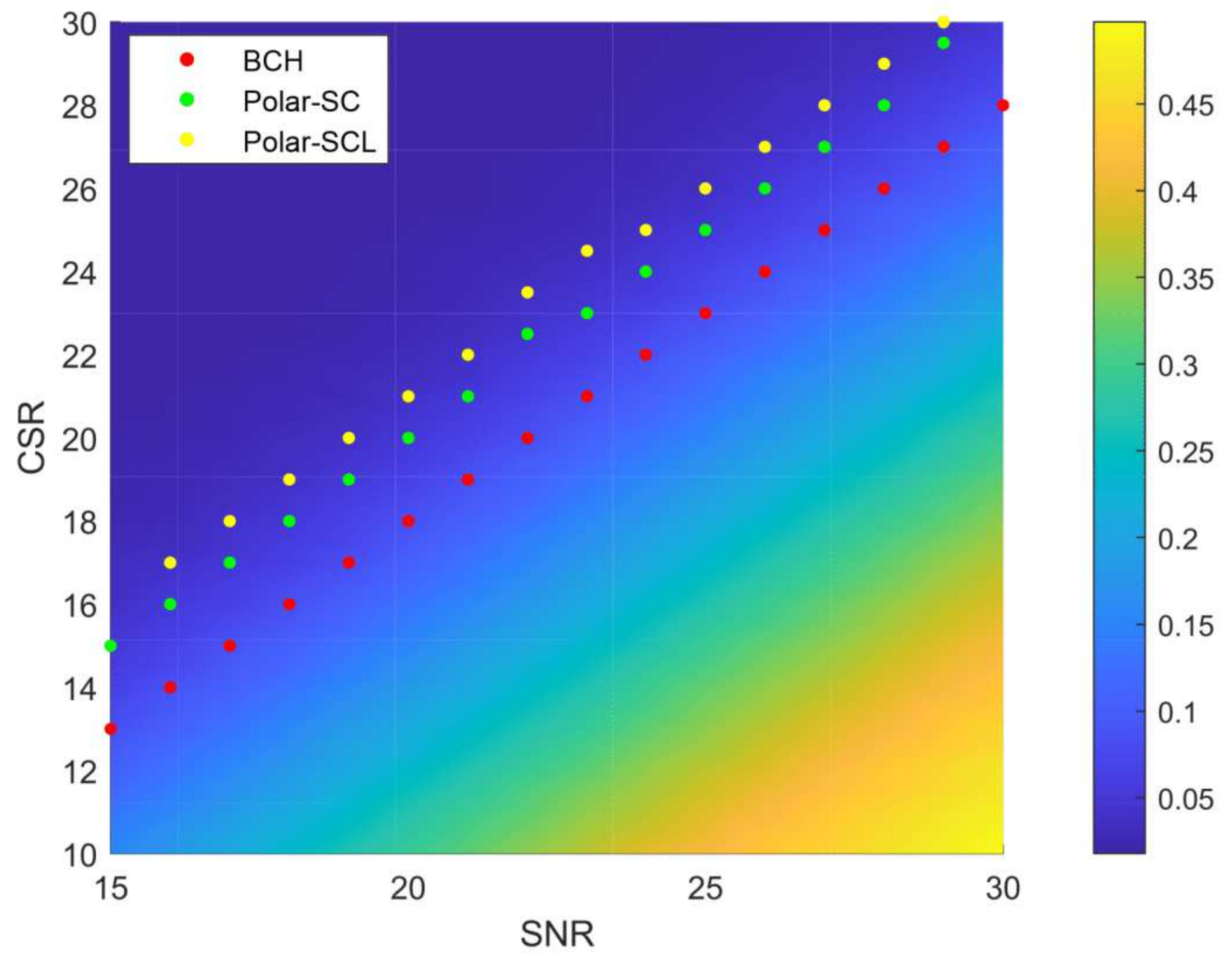

Figure 13

$\mathrm{KL}$ divergence of magnitudes in constellation errors 


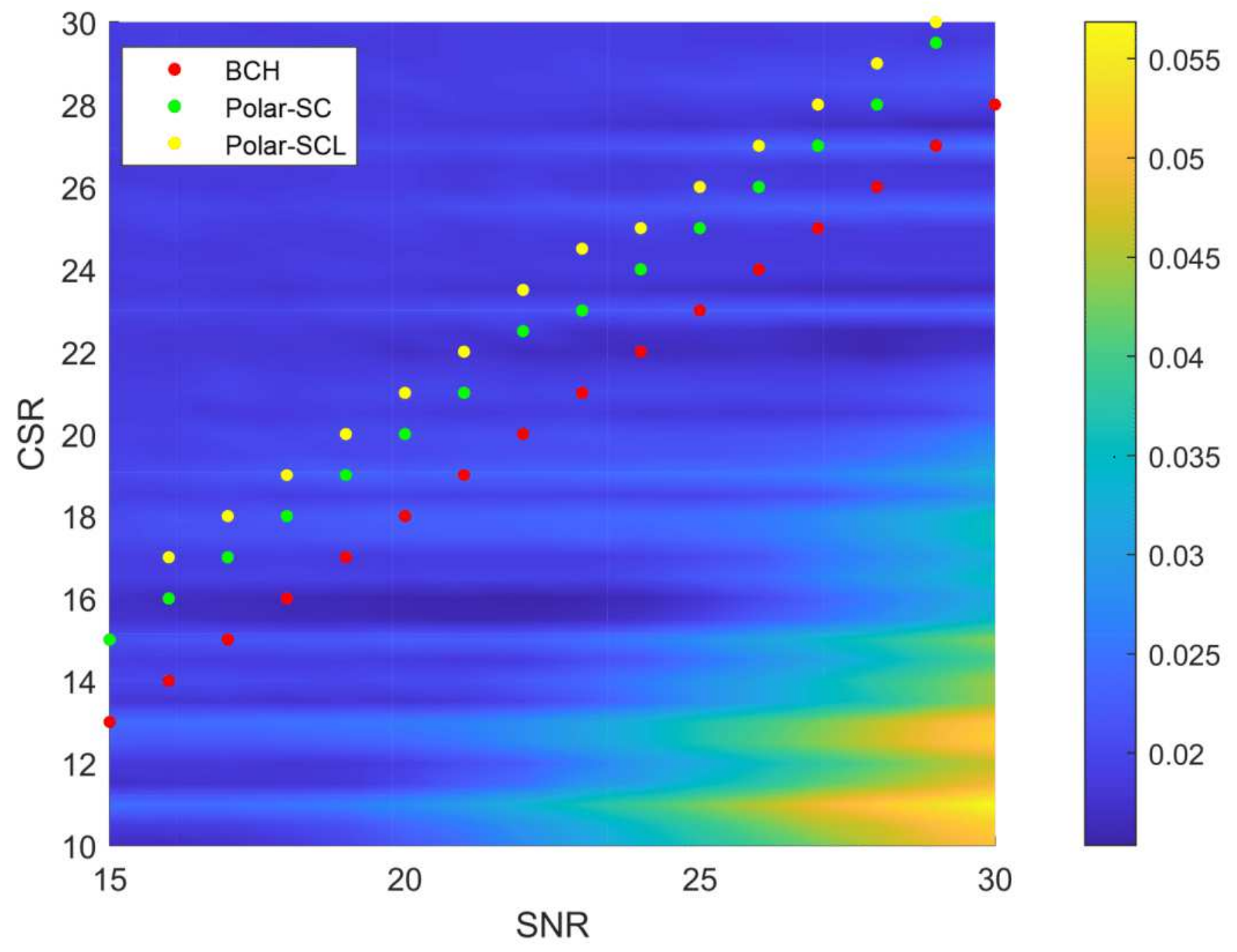

Figure 14

$\mathrm{KL}$ divergence of phase in constellation errors 


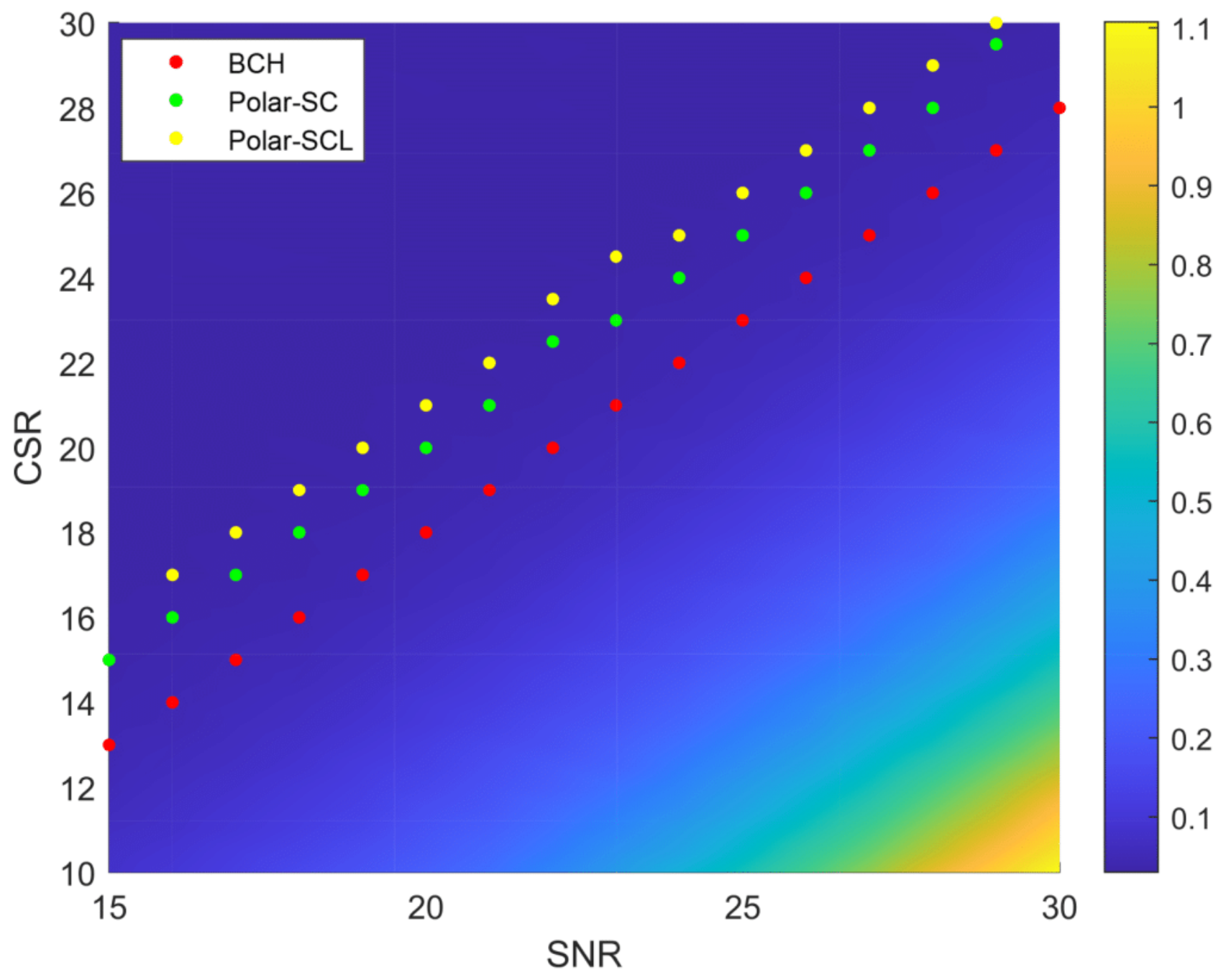

Figure 15

KS distance of I vectors in constellation errors 


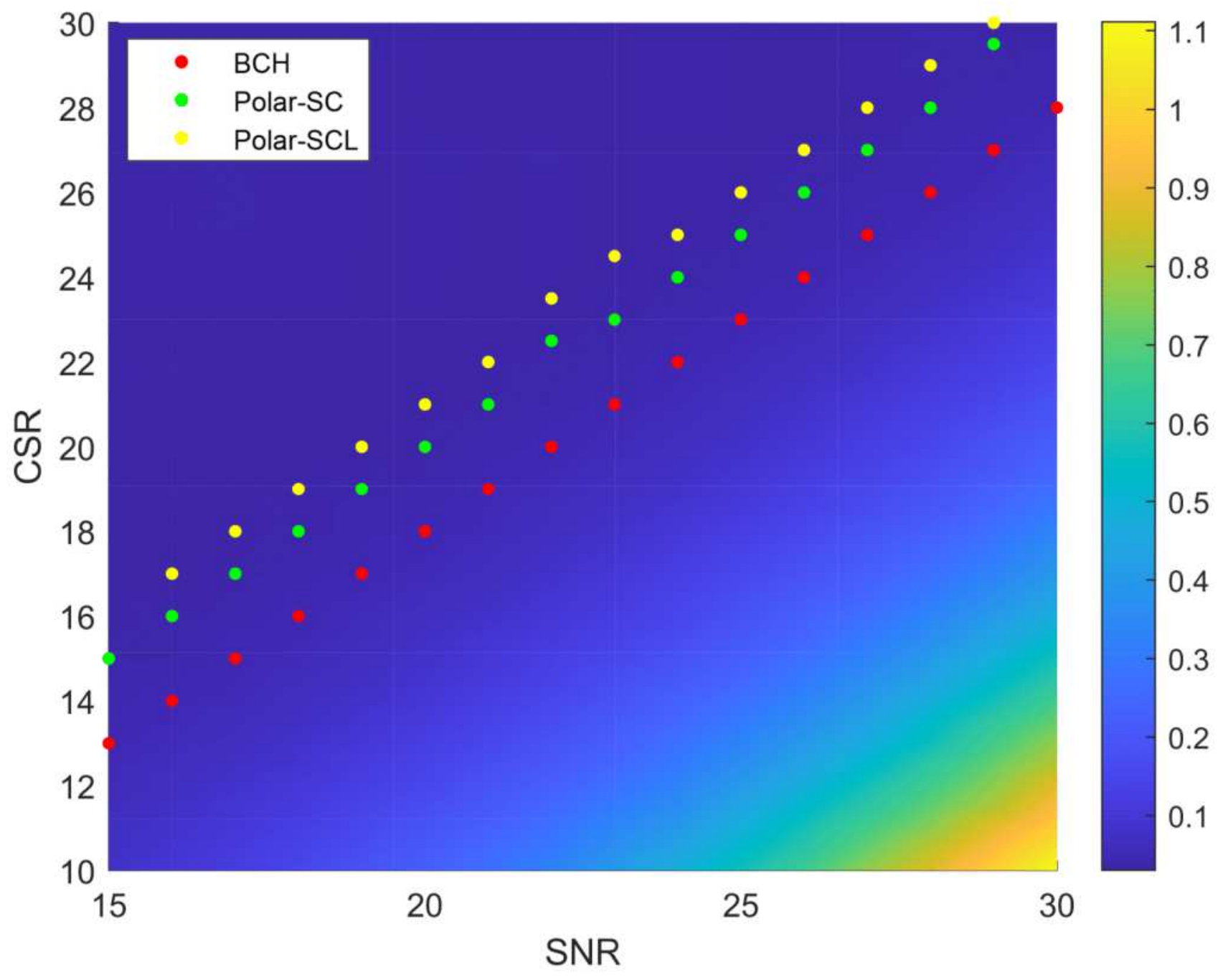

Figure 16

KS distance of Q vectors in constellation errors 


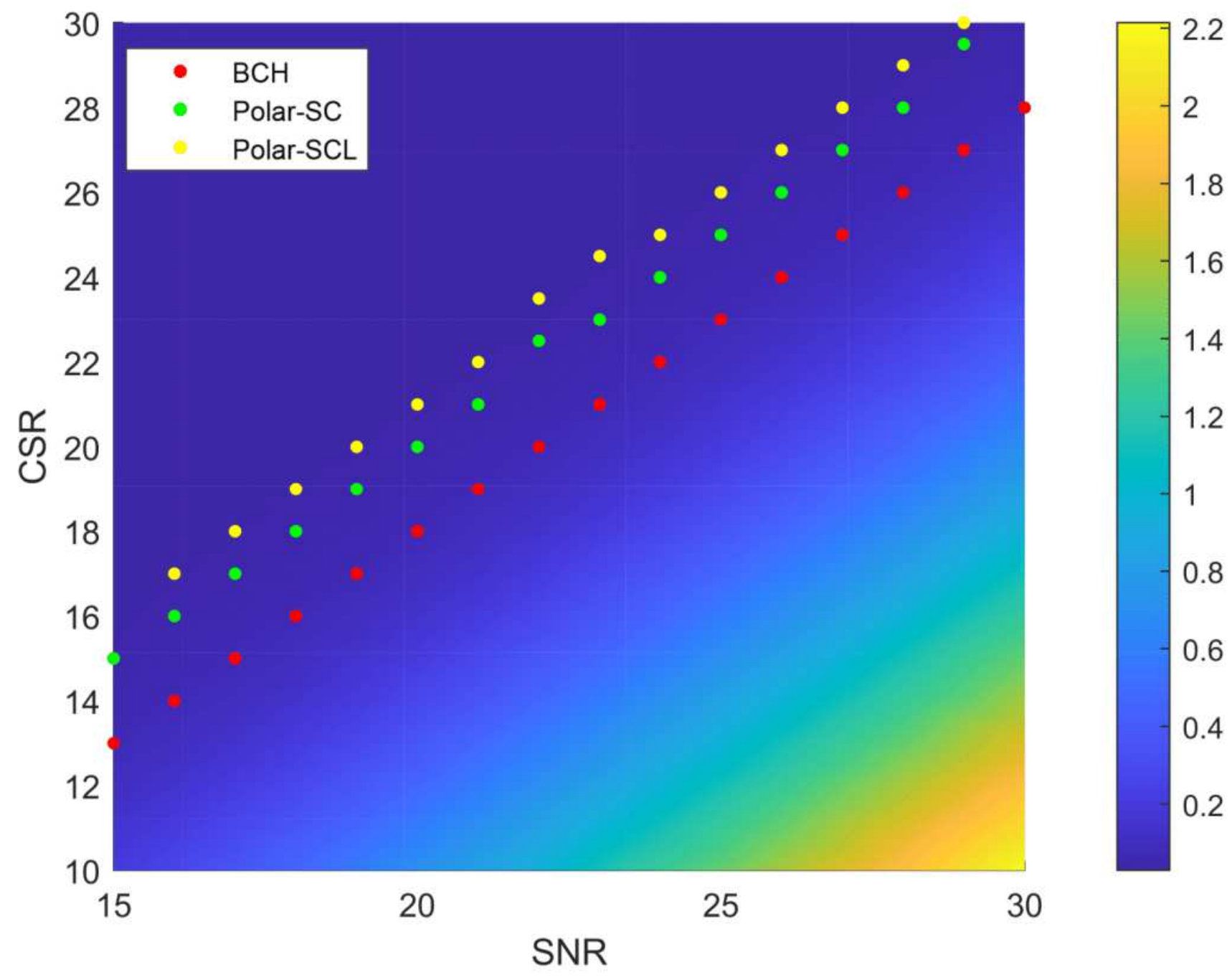

Figure 17

KS distance of magnitudes in constellation errors 


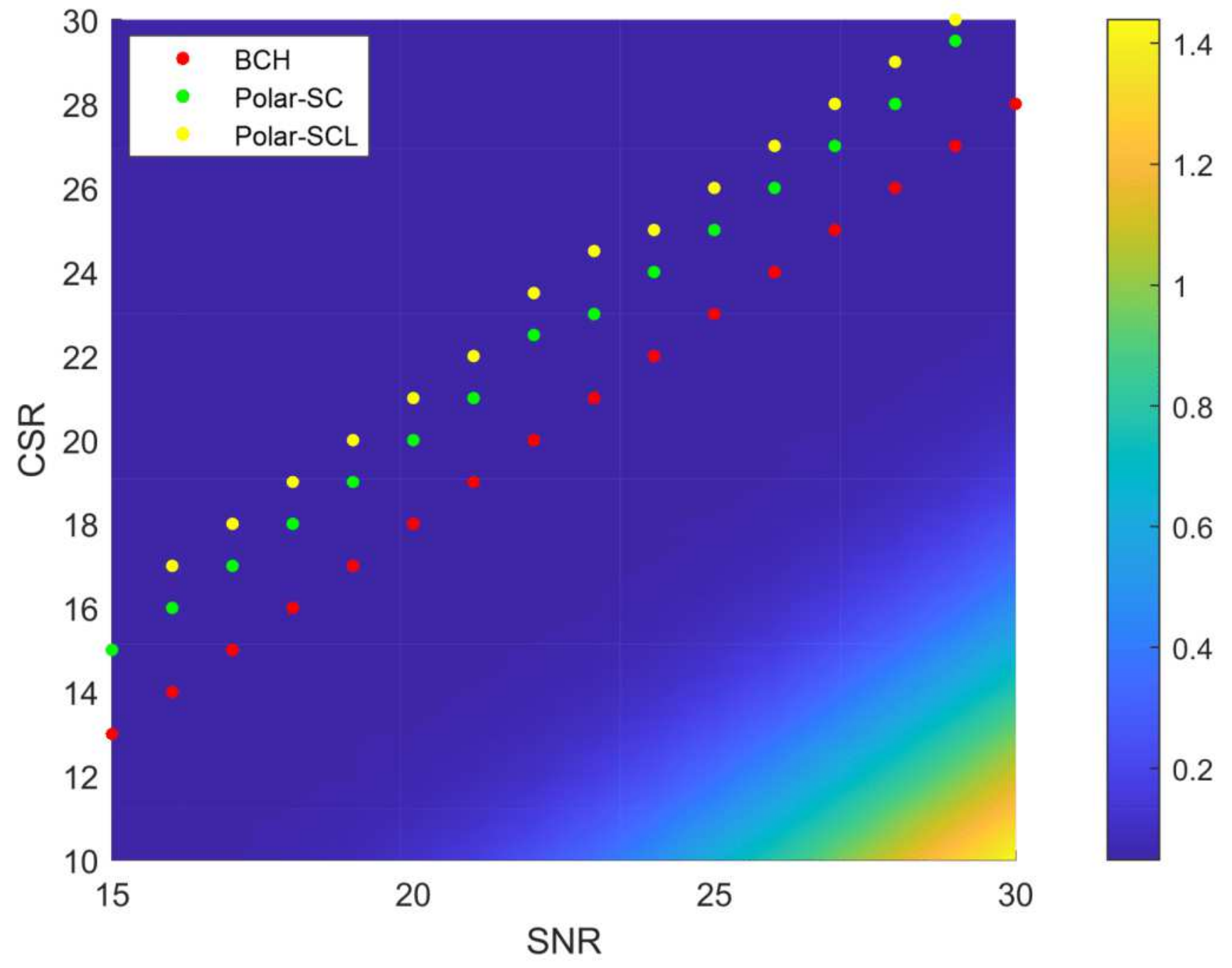

Figure 18

KS distance of phase in constellation errors 